\title{
Type-based termination of recursive definitions
}

\author{
G. BARTHE${ }^{1}$, M. J. FRADE², E. GIMÉNEZ ${ }^{3,4}$, L. PINTO ${ }^{5}$, and T. UUSTALU ${ }^{2,6}$ \\ ${ }^{1}$ INRIA Sophia-Antipolis, 2004 route des Lucioles, BP 93, \\ F-06902 Sophia-Antipolis Cedex, France \\ ${ }^{2}$ Dep. de Informática, Universidade do Minho, Campus de Gualtar, \\ P-4710-057 Braga, Portugal \\ 3 Trusted Logic, 5 rue du Bailliage, F-78000 Versailles, France \\ ${ }^{4}$ Instituto de Computación, Universidad de la República, Julio Herrera y Reissig 565, \\ 11300 Montevideo, Uruguay \\ ${ }^{5}$ Dep. de Matemática, Universidade do Minho, Campus de Gualtar, \\ P-4710-057 Braga, Portugal \\ ${ }^{6}$ Institute of Cybernetics, Akadeemia tee 21, EE-12618 Tallinn, Estonia
}

Received 19 December 2000; revised 7 July 2002

The paper introduces $\lambda$, a simply typed lambda calculus supporting inductive types and recursive function definitions with termination ensured by types. The system is shown to enjoy subject reduction, strong normalization of typable terms and to be stronger than a related system $\lambda_{\mathcal{G}}$ in which termination is ensured by a syntactic guard condition. The system can, at will, be extended to also support coinductive types and corecursive function definitions.

\section{Introduction}

Background Most functional programming languages (ML, Haskell, etc) and proof development systems based on the proofs-as-programs paradigm of logic (COQ, HOL, PVS, etc) rely on powerful type theories featuring inductive types such as natural numbers or lists. Those languages come equipped with a mechanism for recursive definition of functions. However, there are significant differences between the mechanisms used in functional programming languages and in proof development systems.

The first difference concerns the termination of recursive functions. While in functional programming languages recursive functions are allowed to diverge, in proof development systems non-terminating functions must be banished from the language, as they almost always lead to logical paradoxes.

The second difference concerns how recursive definitions are introduced. In functional programming languages, recursive functions are described in terms of a pattern-matching operator (case) and a general fixpoint operator (let-rec). For example, the addition of two natural numbers could be introduced as follows:

let rec plus $\mathrm{n} m=$ 


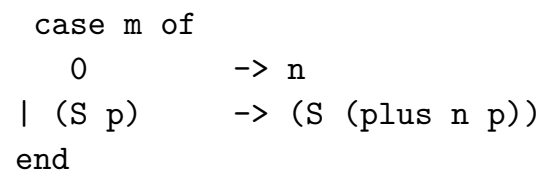

On the other hand, in the traditional presentations of type-based proof development systems (Coquand and Paulin 1990; Dybjer 1994; Luo 1994; Nordström et al. 1990), a recursive function $f: d \rightarrow \theta$ on an inductive type $d$ is defined by means of the elimination rule of $d$, where both pattern matching and recursion are built into a single scheme which ensures termination. In this approach, the function plus can be encoded using the elimination rule of natural numbers nat_elim $\theta: \theta \rightarrow($ Nat $\rightarrow \theta \rightarrow \theta) \rightarrow$ Nat $\rightarrow \theta$, which corresponds to the primitive recursion scheme:

let plus $\mathrm{n} m=$ (nat_elim nat $\mathrm{n}$ (fun $\mathrm{p} \mathrm{r} \rightarrow(\mathrm{S} r)$ ) $\mathrm{m}$ )

This approach is theoretically sound. However practice has shown that eliminators are rather cumbersome to use, whereas case-expressions and fixpoint expressions lead to more concise and readable definitions. Looking for a good compromise between termination and presentation issues, (Coquand 1992) suggested that recursors should be replaced by case-expressions and a restricted form of fixpoint expressions, see also (Giménez 1995). The restriction is imposed through a predicate $\mathcal{G}_{f}$ on untyped terms. This predicate enforces termination by constraining all recursive calls to be applied to terms smaller than the formal argument $x$ of $f$-for instance, a pattern variable issued from a case expression on $x$. The restricted typing rule for fixpoint expressions hence becomes:

$$
\frac{f: \text { Nat } \rightarrow \theta \vdash e: \text { Nat } \rightarrow \theta}{\vdash(\text { letrec } f=e): \text { Nat } \rightarrow \theta} \quad \text { if } \mathcal{G}_{f}(e)
$$

This alternative approach, called guarded by destructors recursion in (Giménez 1995), has been implemented in the COQ system. Five years of experiments carried out with CoQ have shown that it actually provides much more palatable representations of recursive functions.

However, the use of an external predicate $\mathcal{G}$ on untyped terms suffers from several weaknesses:

1 The guard predicate is too syntax-sensitive and too weak.

The acceptance of a recursive definition becomes too sensitive to the syntactical shape of its body. Sometimes, a small change in the definition could make it to no longer satisfy the guardedness condition. As an example, consider the following modification of the plus function, where the condition is no longer satisfied because of the introduction of a redex in the definition:

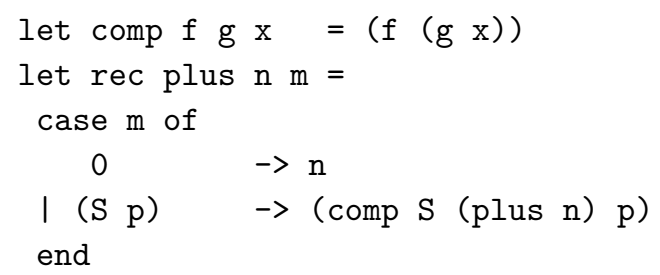

In addition, the guard predicate rejects many terminating recursive definitions such 
as the Euclidean division, Ackermann's function, or functions that swap arguments, such as subtyping algorithms for higher-order languages

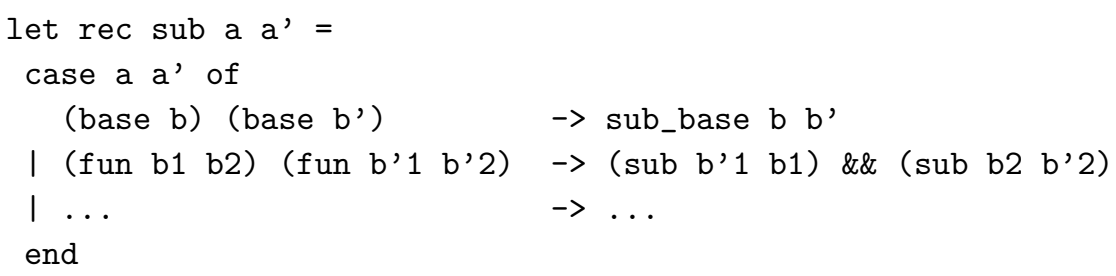

2 The guard predicate is hard to implement and hard to extend.

The guardedness condition is among the main sources of bugs in the implementation of the proof system. In order to improve the number of definitions accepted by the system, the guardedness condition has become more and more complicated hence prone to errors.

Besides, it is easier to extend the type system than to extend the guardedness condition: type conditions are expressed as local constraints associated to each construction of the language whereas the guard predicate yields global constraints.

3 The guard predicate is often defined on normal forms.

Often the guard predicate is defined on normal forms only, which renders the typing rule $(*)$ useless in practice. Subsequently, the typing rule $(*)$ is usually replaced by the more liberal typing rule

$$
\frac{f: \text { Nat } \rightarrow \theta \vdash e: \text { Nat } \rightarrow \theta}{\vdash(\text { letrec } f=e): \text { Nat } \rightarrow \theta} \quad \text { if } \mathcal{G}_{f}(\text { nf } e)
$$

where $\mathrm{nf}$ is the partial function associating to an expression its normal form. Now the modified rule introduces two further complications:

(a) The new guard condition leads to inefficient type-checking.

Verifying the guardedness condition makes type-checking less efficient as the body of a recursive definition has to be reduced for being checked - expanding previously defined constants like the constant comp in the example above.

(b) The new guard condition destroys strong normalization.

For example, the normal form of the following definition satisfies the guardedness condition, but not the definition itself:

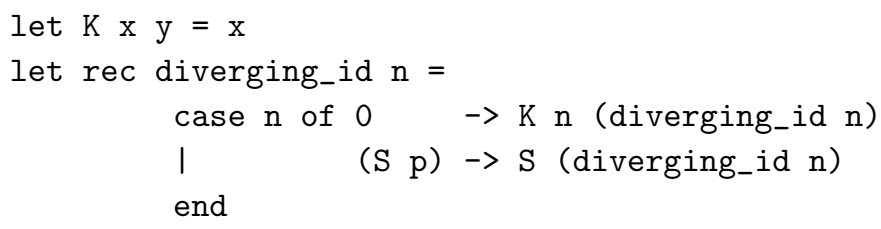

There is an infinite reduction sequence for the term diverging_id $0:^{\dagger}$

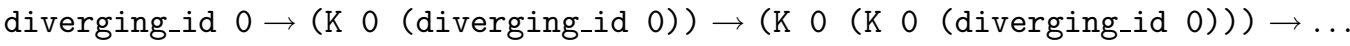

One solution around this problem (the solution has been considered for CoQ) is

$\dagger$ In fact, CoQ 7.1 accepts this definition of diverging_id! 
to store recursive definitions with their bodies in normal forms, as enforced by the rule

$$
\frac{f: \text { Nat } \rightarrow \theta \vdash e: \text { Nat } \rightarrow \theta}{\vdash(\text { letrec } f=(\text { nf } e)): \text { Nat } \rightarrow \theta} \quad \text { if } \mathcal{G}_{f}(\text { nf } e)
$$

but the rule has severe drawbacks: (1) proof terms become huge; (2) the expressions being stored are not those constructed interactively by the user; (3) the modified typing rule for fixpoint expressions is not syntax-directed, i.e. one cannot guess the expression $e$ appearing in the premise from the conclusion of the rule.

In order to circumvent those weaknesses, some authors have proposed semantically motivated type systems that ensure the termination of recursive definitions through typing (Giménez 1998; Amadio and Coupet-Grimal 1998; Barras 1999). The idea, which already occurs in Mendler's work (Mendler 1991), consists in regarding an inductive type $d$ as the least fixpoint of a monotonic operator $\widehat{-}^{d}$ on types, and to enforce termination of recursive functions by requiring that the definition of $f: \widehat{\alpha}^{d} \rightarrow \theta$, where $\alpha$ may be thought as a subtype of $d$, only relies on structurally smaller function calls, embodied by a function $f_{\text {ih }}: \alpha \rightarrow \theta$. This approach to terminating recursion, which we call type-based, offers several advantages over the guarded by destructors approach. In particular, it addresses all the above-mentioned weaknesses.

This article The purpose of this paper is to introduce $\lambda$, a simply typed $\lambda$-calculus that supports type-based recursive definitions. Although heavily inspired from previous work by Giménez (Giménez 1998) and closely related to recent work by Amadio and Coupet (Amadio and Coupet-Grimal 1998), the technical machinery behind our system puts a slightly different emphasis on the interpretation of types. More precisely, we formalize the notion of type-based termination using a restricted form of type dependency (a.k.a. indexed types), as popularized by (Xi and Pfenning 1998; Xi and Pfenning 1999). This leads to a simple and intuitive system which is robust under several extensions, such as mutually inductive datatypes and mutually recursive function definitions; however, such extensions are not treated in the paper.

The basic idea is to proceed as follows:

- First, every datatype $d$ is replaced by a family of approximations indexed over a set of stages, which are used to record a bound on the "depth" of values. Here, we adopt a simple minded approach and let stages range over the syntax

$$
s:=\imath|\widehat{s}| \infty
$$

where $\imath$ ranges over stage variables, the hat operator $\widehat{-}$ is a function mapping a stage to its "successor" and $\infty$ is the stage at which the iterative approximation process converges to the datatype itself.

- Second, a recursive definition of a function, say $f: d \rightarrow \theta$ should be given by a term $e$ constructing a function $g^{\prime}: d^{\imath} \rightarrow \theta$ from $g: d^{\imath} \rightarrow \theta$, where $\imath$ ranges over stages (in other words, $e$ should be stage-polymorphic).

In order to illustrate the machinery involved, let us consider the inductive type Nat 
whose constructors are $\mathrm{o}:$ Nat and $\mathrm{s}: \mathrm{Nat} \rightarrow$ Nat. The typing rules are

$$
\overline{\vdash \mathrm{o}: \mathrm{Nat}^{\widehat{s}}} \frac{\vdash n: \mathrm{Nat}^{s}}{\vdash \mathrm{s} n: \mathrm{Nat}^{\widehat{s}}}
$$

and, as an instance of the subsumption rule,

$$
\frac{\vdash n: \mathrm{Nat}^{s}}{\vdash n: \mathrm{Nat}^{\widehat{s}}}
$$

Finally recursive functions from Nat to $\theta$ are constructed with the following typing rule:

$$
\frac{f: \mathrm{Nat}^{2} \rightarrow \theta \vdash e: \mathrm{Nat}^{\hat{\imath}} \rightarrow \theta}{\vdash(\text { letrec } f=e): \mathrm{Nat} \rightarrow \theta}
$$

where $\imath$ is fresh wrt. $\theta$. As shall be shown later, such recursive functions are terminating and, despite its simplicity, this mechanism is powerful enough to capture course-ofvalue primitive recursion. Conformance to the scheme and hence termination is enforced through types.

Organization The remainder of this paper is organized as follows. In Section 2, we present the system $\lambda$ formally. In Section 3 , we show that $\lambda$ is well-behaved, and in particular enjoys subject reduction and strong normalisation. In Section 4 , we introduce $\lambda_{\mathcal{G}}$ and prove that $\lambda^{\wedge}$ strictly extends the system $\lambda_{\mathcal{G}}$. In Section 5 , we consider an extension of $\lambda^{\wedge}$ with coinductive types. We review related work in Section 6 and conclude in Section 7 .

\section{The System $\lambda$ 个}

In this section, we introduce $\lambda$, a simply typed lambda calculus featuring strongly positive, finitely iterated parametric inductive types (in the sense of, e.g., (Martin-Löf 1971)) and type-based termination of recursive definitions. The calculus is à la Curry: terms come without any type annotations.

\subsection{Datatypes, constructors}

Datatypes and constructors are named: we assume given two denumerable sets $\mathcal{D}$ of datatype identifiers and $\mathcal{C}$ of constructor identifiers. On datatypes, we assume a stratification that ensures that the dependency relation between datatypes is well-founded. Hence each datatype $d$ is assigned a $\operatorname{stratum} \operatorname{str}(d) \in \mathbb{N}$. Datatypes and constructors may only accept a fixed number of arguments, so we stipulate that every datatype identifier $d$ (resp. constructor $c$ ) has a fixed arity $\operatorname{ar}(d) \in \mathbb{N}($ resp. $\operatorname{ar}(c) \in \mathbb{N})$ that indicates the number of parameters taken by $d$ (resp. $c$ ). Finally, we require that every datatype $d \in \mathcal{D}$ comes equipped with a vector $\mathrm{C}(d) \subseteq \mathcal{C}$ of constructors, and if $d \neq d^{\prime}$ then $\mathrm{C}(d) \cap \mathrm{C}\left(d^{\prime}\right)=\emptyset$.

For the sake of clarity, we adopt the following naming conventions: $d, d^{\prime}, d_{i}, \ldots$ range over $\mathcal{D}$ and $c, c^{\prime}, c_{i}, \ldots$ range over $\mathcal{C}$. 


\subsection{Terms and reduction}

Terms are built from variables, abstractions, applications, constructors, case-expressions and recursive definitions. Assume we have a denumerable set $\mathcal{V}_{\mathcal{E}}$ of (object) variables, and let $x, x^{\prime}, x_{i}, y, \ldots$ range over $\mathcal{V}_{\mathcal{E}}$.

Notation 2.1. For every set $A$, we let $A^{*}$ denote the set of lists over $A$, and \langle\rangle denote the empty list. $\vec{a}$ range over $A^{*}$ if $a$ ranges over $A$. $\# \vec{a}$ denotes the length of $\vec{a}$, and $\vec{a}[i]$ denotes, when it exists, the $i$ th element of $\vec{a}$. For convenience, we will sometimes write lists in the form $\left\langle a_{1}, \ldots, a_{n}\right\rangle$ instead of $a_{1} \ldots a_{n}$.

Definition 2.2 (Terms). The set $\mathcal{E}$ of terms is given by the abstract syntax

$$
e, e^{\prime}::=x|\lambda x . e| e e^{\prime}|c| \text { case } e^{\prime} \text { of }\{\vec{c} \Rightarrow \vec{e}\} \mid(\text { letrec } x=e)
$$

where in the clause for case-expressions it is assumed that $\vec{c}=\mathrm{C}(d)$ for some $d \in \mathcal{D}$.

Free and bound variables, substitution, etc. are defined as usual. We let $e\left[x:=e^{\prime}\right]$ be the result of replacing all free occurrences of $x$ in $e$ with $e^{\prime}$.

The reduction calculus is given by $\beta$-reduction for function application, $\iota$-reduction for case analysis and $\mu$-reduction for unfolding recursive definitions, which is only allowed in the context of application to a constructor application.

\section{Definition 2.3 (Reduction Calculus).}

$1 \beta$-reduction $\rightarrow \beta$ is defined as the compatible closure of the rule

$$
(\lambda x . e) e^{\prime} \rightarrow \beta \quad e\left[x:=e^{\prime}\right]
$$

$2 \iota$-reduction $\rightarrow_{\iota}$ is defined as the compatible closure of the rule

$$
\text { case }\left(c_{i} \vec{a}\right) \text { of }\left\{c_{1} \Rightarrow e_{1}|\ldots| c_{n} \Rightarrow e_{n}\right\} \rightarrow_{\iota} e_{i} \vec{a}
$$

where $\# \vec{a}=\operatorname{ar}\left(c_{i}\right)$.

$3 \mu$-reduction $\rightarrow \mu$ is defined as the compatible closure of the rule

$$
(\text { letrec } f=e)(c \vec{a}) \rightarrow_{\mu} e[f:=(\text { letrec } f=e)](c \vec{a})
$$

where $\# \vec{a}=\operatorname{ar}(c)$.

$4 \quad \beta \iota \mu$-reduction $\rightarrow \beta \iota \mu$ is defined as $\rightarrow_{\beta} \cup \rightarrow_{\iota} \cup \rightarrow_{\mu}$.

Remark 2.4. In the formulation of the $\beta$ - and $\mu$-reduction rules, we rely on a variable convention: in the $\beta$-rule, the bound variables of $e$ are assumed to be different from the free variables of $e^{\prime}$; in the $\mu$-rule, the bound and the free variables of $e$ are assumed to be different.

The mechanics of the reduction calculus is illustrated by the following example.

Example 2.5. Consider the inductive type of natural numbers Nat with $C(N a t)=\{0, s\}$. Let plus $\equiv\left(\right.$ letrec plus $=\lambda x$. $\lambda y$. case $x$ of $\left\{\mathrm{o} \Rightarrow y \mid \mathrm{s} \Rightarrow \lambda x^{\prime}\right.$. s (plus $\left.\left.\left.x^{\prime} y\right)\right\}\right)$. The following is a reduction sequence that computes one plus two, where as usual $\rightarrow_{\beta}$ denotes the reflexive and transitive closure of $\rightarrow \beta$. 


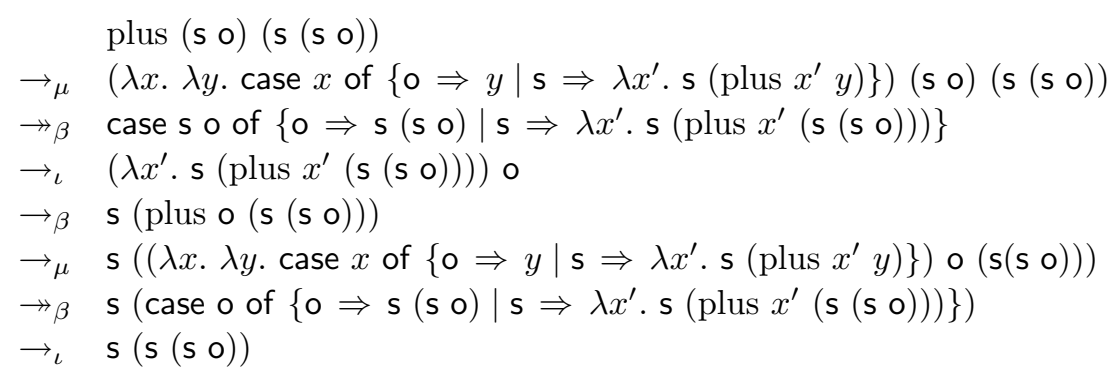

\subsection{Types and typing system}

Assume now given two denumerable sets $\mathcal{V}_{\mathcal{T}}$ of type variables and $\mathcal{V}_{\mathcal{S}}$ of stage variables. Adopt the naming conventions that $\alpha, \alpha^{\prime}, \alpha_{i}, \beta, \delta, \ldots$ range over $\mathcal{V}_{\mathcal{T}}$ and $\imath, \jmath, \ldots$ range over $\mathcal{V}_{\mathcal{S}}$. Proceeding from these, we define stage and type expressions. Stage expressions are built of stage variables, a symbol for the successor function on stages, and a symbol for the limit stage. A type expression is either a type variable, a function type expression or a datatype approximation expression.

\section{Definition 2.6 (Stages and types).}

1 The set $\mathcal{S}$ of stage expressions is given by the abstract syntax:

$$
s, r::=\imath|\infty| \widehat{s}
$$

2 The set $\mathcal{T}$ of type expressions is given by the abstract syntax:

$$
\sigma, \tau::=\alpha|\tau \rightarrow \sigma| d^{s} \vec{\tau}
$$

where in the last clause, it is assumed that the length of $\vec{\tau}$ is exactly $\operatorname{ar}(d)$.

Notation 2.7. Very often we write $\vec{\tau} \rightarrow \sigma$ as an abbreviation for $\tau_{1} \rightarrow \ldots \rightarrow \tau_{n} \rightarrow \sigma$, and $d \vec{\sigma}$ as an abbreviation for $d^{\infty} \vec{\sigma}$.

In order to present the typing rules for constructor and case expressions, we have to have a means for fixing the intended typings of the constructors. To this end, we introduce notions of constructor scheme and constructor scheme instantiation.

Definition 2.8 (Constructor scheme). A constructor scheme is a triple $(\delta, \vec{\alpha}, \vec{\sigma})$ where $\delta, \vec{\alpha} \in \mathcal{V}_{\mathcal{T}}$ and $\vec{\sigma} \in \mathcal{T}$ such that

1 each $\sigma_{i}$ is positive w.r.t. $\delta$, see Figure 5;

2 each $\sigma_{i}$ is positive w.r.t. each $\alpha_{j}$, see Figure 5;

$3 \vec{\alpha}, \delta$ are pairwise distinct;

$4 \vec{\alpha}, \delta$ are the only type variables that can occur in $\vec{\sigma}$;

5 there are no occurrences of stage variables in $\vec{\sigma}$.

The set of constructor schemes is denoted by $\mathcal{C S}$.

Observe that type parameters have to appear only positively in the argument types of the constructors. This makes it possible to parameterize the type of lists with respect 
to the type of elements, binary trees with respect to the type of node labels, arbitrarily branching trees with respect to the type of node labels, but not with respect to the branching type.

In the sequel, we assume given a map $D: \mathcal{C} \rightarrow \mathcal{C S}$ that respects arities: formally, for every datatype $d$ and $c \in \mathrm{C}(d)$,

$$
\mathrm{D}(c)=(\delta, \vec{\alpha}, \vec{\sigma}) \quad \text { with } \quad \# \vec{\alpha}=\operatorname{ar}(d) \text { and } \# \vec{\sigma}=\operatorname{ar}(c)
$$

This mapping has to satisfy the following condition: if $c \in \mathrm{C}(d)$ and $\mathrm{D}(c)=(\delta, \vec{\alpha}, \vec{\sigma})$, then any $d^{\prime} \in \mathcal{D}$ appearing in $\vec{\sigma}$ satisfies $\operatorname{str}\left(d^{\prime}\right)<\operatorname{str}(d)$. This ensures that only finitely iterated inductive definitions are permitted (excluding mutual induction) and is made use of in the model construction (Definition 3.23) in the proof of strong normalization.

Constructor schemes specify the possible typings for the arguments of each given constructor of every possible datatype: if $\delta$ is an approximation of the datatype, and $\vec{\alpha}$ are the parameters of the datatype, then $\vec{\sigma}$ is a possible typing for the arguments of the constructor.

Example 2.9. Consider Bool, Nat, List, Tree, Ord $\in \mathcal{D}$. We have

$$
\begin{array}{ll}
\mathrm{C}(\text { Bool })=\{\text { true }, \text { false }\} & \mathrm{D}(\text { true })=(\delta,\langle\rangle,\langle\rangle) \\
& \mathrm{D}(\text { false })=(\delta,\langle\rangle,\langle\rangle)
\end{array}
$$

for the datatype of booleans;

$$
\begin{aligned}
& \mathrm{C}(\mathrm{Nat})=\{\mathrm{o}, \mathrm{s}\} \\
& \mathrm{D}(\mathrm{o})=(\delta,\langle\rangle,\langle\rangle) \\
& \mathrm{D}(\mathrm{s})=(\delta,\langle\rangle,\langle\delta\rangle)
\end{aligned}
$$

for the datatype of natural numbers;

$$
\begin{array}{ll}
\mathrm{C}(\text { List })=\{\text { nil, cons }\} & \mathrm{D}(\text { nil }) \\
& \mathrm{D}(\text { cons })=(\delta,\langle\alpha\rangle,\langle\rangle) \\
&
\end{array}
$$

for lists;

$$
\mathrm{C}(\text { Tree })=\{\text { branch }\} \quad \mathrm{D}(\text { branch })=(\delta,\langle\alpha\rangle,\langle\alpha, \text { List } \delta\rangle)
$$

for finitely branching trees; and

$$
\begin{aligned}
& \mathrm{D}(\text { zero })=(\delta,\langle\rangle,\langle\rangle) \\
& \mathrm{C}(\text { Ord })=\{\text { zero, succ, } \lim \} \quad \mathrm{D}(\text { succ })=(\delta,\langle\rangle,\langle\delta\rangle) \\
& \mathrm{D}(\lim )=(\delta,\langle\rangle,\langle\text { Nat } \rightarrow \delta\rangle)
\end{aligned}
$$

for ordinals (or better said, for ordinal notations).

Each particular legal typing for the arguments of a constructor is obtained by instantiating the associated constructor scheme. The concept of instance of a constructor scheme is formally defined as follows.

Definition 2.10 (Instance). Let $d \in \mathcal{D}, c \in \mathrm{C}(d), s \in \mathcal{S}$ and $\vec{\tau} \in \mathcal{T}$ such that $\# \vec{\tau}=\operatorname{ar}(d)$. Assume $\mathrm{D}(c)=(\delta, \vec{\alpha}, \vec{\sigma})$. An instance of $c$ w.r.t. $s$ and $\vec{\tau}$ is defined as follows

$$
\text { Inst }_{c}^{s} \vec{\tau}=\vec{\sigma}\left[\delta:=d^{s} \vec{\tau}\right][\vec{\alpha}:=\vec{\tau}]
$$


We now turn to the typing system. On the stages, we introduce a comparison relation. Importantly, the stage comparison rules state that all stages beyond the limiting stage are equivalent. On top of the stage comparison relation, another set of rules defines a subtyping relation on types. A crucial fact stated by these rules is that a given approximation of a datatype is always included in the next one.

Definition 2.11 (Stage comparison and subtyping). $\quad \tau$ is a subtype of $\sigma$, written $\tau \sqsubseteq \sigma$, is defined by the rules of Figure 2 , where $s \preccurlyeq r$ is defined by the rules of Figure 1 .

$$
\text { (refl) } \overline{s \preccurlyeq s} \quad \text { (trans) } \quad \frac{s \preccurlyeq r \quad r \preccurlyeq p}{s \preccurlyeq p} \quad \text { (hat) } \quad \overline{s \preccurlyeq \widehat{s}} \quad \text { (infty) } \quad \overline{s \preccurlyeq \infty}
$$

Fig. 1. Stage comparison rules

$$
\text { (refl) } \frac{}{\sigma \sqsubseteq \sigma} \quad \text { (data) } \frac{s \preccurlyeq r \quad \tau_{i} \sqsubseteq \tau_{i}^{\prime} \quad(1 \leq i \leq \operatorname{ar}(d))}{d^{s} \vec{\tau} \sqsubseteq d^{r} \overrightarrow{\tau^{\prime}}} \quad \text { (func) } \quad \frac{\tau^{\prime} \sqsubseteq \tau \quad \sigma \sqsubseteq \sigma^{\prime}}{\tau \rightarrow \sigma \sqsubseteq \tau^{\prime} \rightarrow \sigma^{\prime}}
$$

Fig. 2. Subtyping rules

Notation 2.12. We write $\vec{\sigma} \sqsubseteq \vec{\tau}$, if $\# \vec{\sigma}=\# \vec{\tau}$ and $\sigma[i] \sqsubseteq \tau[i]$ for $i=1 . . \# \vec{\sigma}$.

Lemma 2.13. If $\sigma \sqsubseteq \tau$ and $\tau \sqsubseteq \theta$, then $\sigma \sqsubseteq \theta$.

Lemma 2.14. If $\widehat{r} \preccurlyeq \widehat{s}$, then $r \preccurlyeq s$.

Proof. By induction on the proof of $p \preccurlyeq \widehat{s}$, one can show that $p \preccurlyeq \widehat{s}$ implies $p \preccurlyeq s$ or $p=\widehat{s}$ from where the claim can be inferred by instantiating $p=\widehat{r}$.

Lemma 2.15. If $r \preccurlyeq s$, then $\operatorname{Inst}_{c}^{r} \vec{\tau} \sqsubseteq \operatorname{Inst}_{c}^{s} \vec{\tau}$.

In order to define the typing relation between terms and type expressions, we need the concepts of context and judgment.

\section{Definition 2.16 (Contexts and judgments).}

1 A context is a finite sequence $x_{1}: \sigma_{1}, \ldots, x_{n}: \sigma_{n}$ where $x_{1}, \ldots, x_{n}$ are pairwise disjoint (object) variables and $\sigma_{1}, \ldots, \sigma_{n}$ are types.

2 A typing judgment is a triple of the form $\Gamma \vdash e: \sigma$, where $\Gamma$ is a context, $e$ is a term and $\sigma$ is a type expression.

The definition of the typing relation itself depends on that of subtyping.

\section{Definition 2.17 (Typing).}

1 A typing judgment is derivable if it can be inferred from the rules of Figure 3 where the positivity condition $\imath$ pos $\sigma$ in the (rec) rule is defined in Figure 4. 


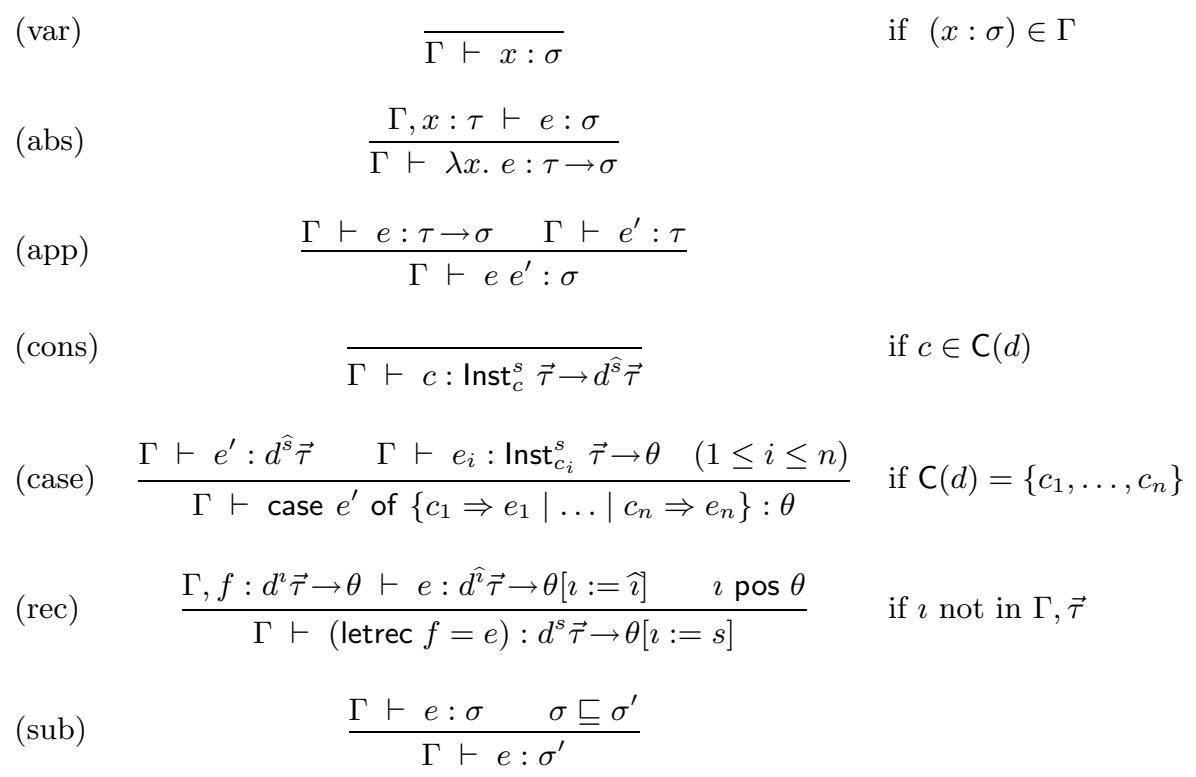

Fig. 3. Typing rules for $\lambda^{\wedge}$

2 A term $e \in \mathcal{E}$ is typable if $\Gamma \vdash e: \sigma$ is derivable for some context $\Gamma$ and type $\sigma$.

$\begin{array}{cccc}(\mathrm{sp} 1) & \frac{(\mathrm{sn} 1)}{\imath \operatorname{pos} \alpha} & \overline{\operatorname{neg} \alpha} \\ (\operatorname{sp} 2) & \frac{\imath \operatorname{neg} \tau \quad \imath \operatorname{pos} \sigma}{\imath \operatorname{pos} \tau \rightarrow \sigma} & (\operatorname{sn} 2) & \frac{\imath \operatorname{pos} \tau \quad \imath \operatorname{neg} \sigma}{\imath \operatorname{neg} \tau \rightarrow \sigma} \\ (\operatorname{sp} 3) & \frac{\imath \operatorname{pos} \tau_{i} \quad(1 \leq i \leq \operatorname{ar}(d))}{\imath \operatorname{pos} d^{s} \vec{\tau}} & (\operatorname{sn} 3) & \frac{\imath \operatorname{nocc} s}{\imath \operatorname{neg} \tau_{i} \quad(1 \leq i \leq \operatorname{ar}(d))} \\ & & \end{array}$

Fig. 4. Positive-negative occurrences of a stage variable

The rules (var), (abs), and (app) come from the standard simply typed $\lambda$-calculus. The rule (sub) is present in any $\lambda$-calculus with subtyping and provides a linkage between the subtyping and typing relations. The remaining rules-(cons), (case) and (rec)-deserve short comments.

The (cons) rule says that applying a constructor of a given datatype to values in an approximation of the datatype gives a value that is guaranteed to be an element in the next approximation. The (case) rule says that the converse is also true: any value in the approximation next to some given one is a result of applying one of the constructors of the datatype to values in the given approximation and can therefore be subjected to case analysis. The (letrec) rule, finally, says that any systematic way of extending a function 


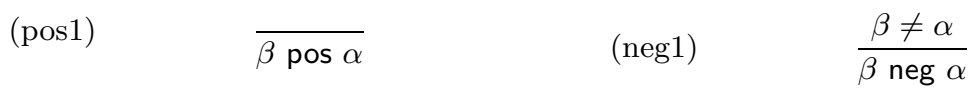

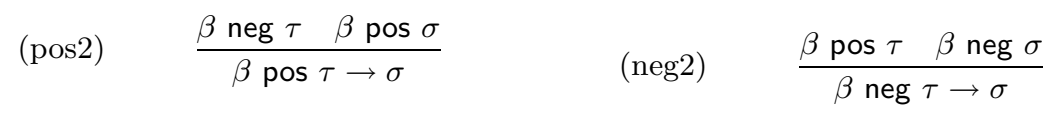

$$
\begin{aligned}
& (\operatorname{pos} 3) \quad \frac{\beta \operatorname{pos} \tau_{i} \quad(1 \leq i \leq \operatorname{ar}(d))}{\beta \operatorname{pos} d^{s} \vec{\tau}} \quad \text { (neg3) } \frac{\beta \operatorname{neg} \tau_{i} \quad(1 \leq i \leq \operatorname{ar}(d))}{\beta \operatorname{neg} d^{s} \vec{\tau}}
\end{aligned}
$$

Fig. 5. Positive-negative occurrences of a type variable

defined on a given approximation of a datatype to work also on the next approximation induces a function defined on the whole datatype, the limit of the approximations. The premiss of this rule involves an implicit universal quantification over the set of all stages (freshness condition!).

\subsection{Some examples}

In order to illustrate the mechanics and expressive power of our calculus, we now give a few examples of programming. We start from simple recursive definitions that are definable in all useful existing systems.

\section{Example 2.18 (Standard examples).}

- The addition of two natural numbers. The only recursive call in this program is made on a depth-one recursive component of the argument value.

$$
\begin{aligned}
& \text { plus } \equiv\left(\text { letrec } \quad \text { plus }_{: \mathrm{Nat}^{2} \rightarrow \mathrm{Nat} \rightarrow \mathrm{Nat}}=\right. \\
& \lambda x_{: \text {Nat }^{\hat{\imath}}} . \lambda y: \text { Nat } \text {. case } x \text { of }\{0 \Rightarrow y \\
& \mathrm{s} \Rightarrow \lambda x_{: \mathrm{Nat}^{2}}^{\prime} \cdot \mathrm{s} \underbrace{\left(\text { plus } x^{\prime} y\right)}_{\text {:Nat }} \\
& ): \quad \quad \mathrm{Nat}^{s} \rightarrow \mathrm{Nat} \rightarrow \mathrm{Nat} \\
& \text { \} }
\end{aligned}
$$

- The concatenation of two lists and the concatenation of a list of lists.

$$
\begin{aligned}
& \text { append } \equiv \quad\left(\text { letrec append }: \text { List }^{2} \tau \rightarrow \text { List } \tau \rightarrow \text { List } \tau=\lambda x_{: \text {List }^{\hat{\imath}} \tau} \cdot \lambda y: \text { List } \tau\right. \text {. } \\
& \text { case } x \text { of }\{\text { nil } \Rightarrow y \\
& \text { cons } \Rightarrow \lambda z_{: \tau} \cdot \lambda x_{: \text {List }^{2} \tau}^{\prime} \text {. cons } z \underbrace{\left(\text { append } x^{\prime} y\right)}_{\text {:List } \tau} \\
& \} \\
& \text { ) : } \quad \text { List }^{s} \tau \rightarrow \text { List } \tau \rightarrow \text { List } \tau
\end{aligned}
$$




$$
\begin{aligned}
& \text { conc } \equiv \quad\left(\text { letrec } \operatorname{conc}_{: \operatorname{List}^{2}(\operatorname{List} \tau) \rightarrow \operatorname{List} \tau}=\lambda x_{: \operatorname{List} \hat{\imath}^{\imath}(\operatorname{List} \tau)} .\right. \\
& \text { case } x \text { of }\{\text { nil } \Rightarrow \text { nil } \\
& \mid \text { cons } \Rightarrow \lambda z_{\text {:List } \tau} \cdot \lambda x_{: \operatorname{List}{ }^{2}(\operatorname{List} \tau)}^{\prime} \text {. append } z \underbrace{\left(\operatorname{conc} x^{\prime}\right)}_{\text {:List } \tau} \\
& \text { \} } \\
& \text { ) : } \quad \operatorname{List}^{s}(\operatorname{List} \tau) \rightarrow \operatorname{List} \tau
\end{aligned}
$$

— The addition of two ordinals.

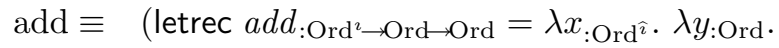

$$
\begin{aligned}
& \text { case } x \text { of } \text { zero } \Rightarrow y \\
& \text { | succ } \Rightarrow \lambda x_{: \operatorname{Ord}^{2}}^{\prime} \text {. succ } \underbrace{\left(a d d x^{\prime} y\right)}_{: \text {Ord }} \\
& \mid \lim \Rightarrow \lambda x_{: \mathrm{Nat} \rightarrow \mathrm{Ord}^{2}}^{\prime} . \lim (\lambda z_{\mathrm{NNat}} . \quad \text { add } \underbrace{\left(x^{\prime} z\right)} y) \\
& \underbrace{\underbrace{\text { Ord }^{2}}_{\text {:Ord }}}_{: \text {Ord }} \\
& \text { ) : } \quad \operatorname{Ord}^{s} \rightarrow \text { Ord } \rightarrow \text { Ord }
\end{aligned}
$$

The following example illustrates the use of subsumption.

Example 2.19 (Example using subsumption). The predicate that decides if a natural number is even or not may be defined as follows. This program involves a recursive call on a deep recursive component of the argument value. To type it, therefore, the subsumption rule has to be used.

$$
\begin{aligned}
& \text { (letrec even }: \mathrm{Nat}^{2} \rightarrow \mathrm{Bool}=\lambda x_{: \mathrm{Nat}^{\hat{\imath}}} \text {. } \\
& \text { case } x \text { of }\{0 \Rightarrow \text { true } \\
& \mathrm{s} \Rightarrow \lambda x_{: \mathrm{Nat}^{2} \sqsubseteq \mathrm{Nat}{ }^{\hat{\imath}}} \text {. case } x^{\prime} \text { of }\{\mathrm{o} \Rightarrow \text { false } \\
& \mid \mathrm{s} \Rightarrow \lambda x_{: \text {Nat }}^{\prime \prime} \cdot \underbrace{\text { even } x^{\prime \prime}}_{: \text {Bool }}\} \\
& \text { ) : } \quad \mathrm{Nat}^{s} \rightarrow \text { Bool }
\end{aligned}
$$

The following examples demonstrate the specific, novel features of $\lambda$. First of all, stages provide a limited means of controlling the effect of a recursively defined function in terms of the relation between the depths of argument and result values.

\section{Example 2.20 (Examples of "exact" typings).}

— The length of a list. This standard program for calculating the length of a list admits 
an unusually "exact" (i.e., tight and therefore informative) type in $\lambda^{\wedge}$.

$$
\begin{aligned}
& \text { length } \equiv \quad \text { (letrec length } \text { List }^{2} \tau \rightarrow \mathrm{Nat}^{2}= \\
& \lambda x_{: \text {List }}{ }^{\hat{\imath}} \tau \text {. case } x \text { of }\{\text { nil } \Rightarrow \circ \\
& \mid \text { cons } \Rightarrow \lambda z_{: \tau} \cdot \lambda x_{: \text {List }^{2} \tau}^{\prime} \cdot \underbrace{\mathrm{s}}_{: \text {Nat }^{\hat{\imath}}} \underbrace{\left(\text { length } x^{\prime}\right)}_{: \text {Nat }^{2}} \\
& \text { ) : } \quad \text { List }^{s} \tau \rightarrow \mathrm{Nat}^{s}
\end{aligned}
$$

- The map of a function on a list. This program is very similar to that for the length function and also admits an "exact" typing, but becomes crucial in an example below.

$$
\begin{aligned}
& \text { map } \equiv \lambda f_{: \tau \rightarrow \sigma} . \quad\left(\text { letrec } m a p_{: \text {List }^{2} \tau \rightarrow \text { List }^{2} \sigma}=\lambda x_{: \text {List }^{\hat{\imath}} \tau} .\right. \\
& \text { case } x \text { of }\{\text { nil } \Rightarrow \text { nil } \\
& \mid \text { cons } \Rightarrow \lambda z_{: \tau} \cdot \lambda x_{: \text {List }^{2} \tau}^{\prime} \cdot \underbrace{\text { cons } \underbrace{(f z)}_{: \text {List }^{2} \sigma}}_{: \text {List }^{\hat{\imath}} \sigma} \\
& \text { \} } \\
& ): \text { List }^{s} \tau \rightarrow \text { List }^{s} \sigma
\end{aligned}
$$

Further, recursive calls are allowed on structurally smaller arguments that cannot be verified to be structurally smaller using a viable syntactic criterion.

\section{Example 2.21 (Examples not handled by the guard condition).}

- The Euclidean division $\left\lceil\frac{x}{y+1}\right\rceil$. This program for the Euclidean division depends on a program for subtraction. It is not typable in systems with a syntactic guard predicate, as, syntactically, (minus $x^{\prime} y$ ) is not properly structurally smaller than $x$ in the program below. In $\lambda$, it is typable because of the "exact" type assignable to minus.

$$
\begin{aligned}
& \text { minus } \equiv \quad\left(\text { letrec minus }: \mathrm{Nat}^{2} \rightarrow \mathrm{Nat}^{\mathrm{N}} \mathrm{Nat}^{2}=\lambda x_{: \mathrm{Nat}^{\hat{\imath}}} \cdot \lambda y_{: \mathrm{Nat}} .\right. \\
& \text { case } x \text { of }\{0 \Rightarrow x \\
& \mathrm{s} \Rightarrow \lambda x_{: \text {Nat }^{2}}^{\prime} \text {. case } y \text { of }\{\mathrm{o} \Rightarrow x \\
& \text { | } \mathrm{s} \Rightarrow \lambda y_{: \text {Nat }}^{\prime} \cdot \underbrace{\text { minus } x^{\prime} y^{\prime}}_{\text {:Nat }^{2}}\} \\
& \text { \} } \\
& \text { ) : } \quad \mathrm{Nat}^{s} \rightarrow \mathrm{Nat} \rightarrow \mathrm{Nat}^{s} \\
& \text { (letrec } \quad \operatorname{div}_{: \mathrm{Nat}^{2} \rightarrow \mathrm{Nat} \rightarrow \mathrm{Nat}^{2}}= \\
& \lambda x_{: \text {Nat }} \hat{\imath} . \lambda y_{\text {Nat }} \text {. case } x \text { of }\{0 \Rightarrow 0 \\
& \mid \mathrm{s} \Rightarrow \lambda x_{: \mathrm{Nat}^{2}}^{\prime} . \mathrm{s} \underbrace{(d i v \underbrace{\left(\operatorname{minus} x^{\prime} y\right)}_{: \mathrm{Nat}^{2}} y)}_{: \mathrm{Nat}^{2}} \\
& ): \quad \mathrm{Nat}^{s} \rightarrow \mathrm{Nat} \rightarrow \mathrm{Nat}^{s}
\end{aligned}
$$

— Flattening of finitely branching trees. This program depends on map and conc. Sim- 
ilarly to div, it is not typable in systems with a syntactic guard predicate.

$$
\begin{aligned}
& \text { (letrec flatten: } \text { Tree }^{2} \tau \rightarrow \text { List } \tau= \\
& \lambda x: \text { Tree }^{\hat{\imath}} \tau \text {. case } x \text { of }\{ \\
& \text { branch } \Rightarrow \lambda z_{: \tau} \cdot \lambda x_{: \text {List }}^{\prime}\left(\text { Tree }^{\imath} \tau\right) \text {. cons } z \underbrace{(\operatorname{conc} \underbrace{\left(\operatorname{map} \text { flatten } x^{\prime}\right)}_{: \text {List }(\text { List } \tau)})}_{: \text {List } \tau} \\
& \text { ) : } \quad \operatorname{Tree}^{s} \tau \rightarrow \text { List } \tau
\end{aligned}
$$

Finally, we give an example demonstrating the usefulness of having the capability of naming stages explicitly: if one recursion is nested in another, we need two distinct free stage variables.

Example 2.22 (Examples involving several stage variables). The Ackermann function. The natural definition of the Ackermann function is not typable in our system. A definition with two recursions, one nesting the other, however, is easy to type.

$$
\begin{aligned}
& \text { ack } \equiv \quad\left(\text { letrec } a c k_{: \mathrm{Nat}^{2} \rightarrow \mathrm{Nat} \rightarrow \mathrm{Nat}}=\lambda x_{: \mathrm{Nat}^{\hat{\imath}}} .\right. \\
& \text { case } x \text { of }\{0 \Rightarrow \underbrace{\lambda z \cdot(\mathrm{s} z)}_{: \text {Nat } \rightarrow \text { Nat }} \\
& \mid \mathrm{s} \Rightarrow \lambda x_{: \mathrm{Nat}^{2}}^{\prime} \text {. (letrec } a c k_{-} x_{: \mathrm{Nat}^{\jmath} \rightarrow \mathrm{Nat}}=\lambda y_{: \mathrm{Nat}^{\hat{\jmath}}} \text {. } \\
& \text { case } y \text { of }\{0 \Rightarrow \underbrace{a c k x^{\prime}(\mathrm{s} o)}_{\text {:Nat }} \\
& \mid \mathrm{s} \Rightarrow \lambda y_{: \text {Nat }^{\jmath}}^{\prime \text { :Nat }} . \underbrace{a c k x^{\prime} \underbrace{\left(a c k \_x y^{\prime}\right)}_{\text {:Nat }}}_{: \text {Nat }}\} \\
& ): \mathrm{Nat} \rightarrow \mathrm{Nat} \\
& \text { ) : } \quad \mathrm{Nat}^{s} \rightarrow \mathrm{Nat} \rightarrow \mathrm{Nat}
\end{aligned}
$$

\section{Meta-theoretical results}

In this section, we prove two important meta-theoretic properties of $\lambda$ - subject reduction and strong normalizability of typable terms.

\subsection{Subject reduction}

The proof of subject reduction for $\lambda$ is quite standard. Before going into this proof, some lemmata involving monotonicity and substitution properties for stages, as well as generation and substitution properties for typing, are considered.

\section{Lemma 3.1 (Generation lemma for subtyping).}

$1 \quad \sigma \sqsubseteq \tau_{1} \rightarrow \tau_{2} \Rightarrow \sigma \equiv \tau_{1}^{\prime} \rightarrow \tau_{2}^{\prime} \wedge \tau_{1} \sqsubseteq \tau_{1}^{\prime} \wedge \tau_{2}^{\prime} \sqsubseteq \tau_{2}$

$2 \quad \tau_{1} \rightarrow \tau_{2} \sqsubseteq \sigma \Rightarrow \sigma \equiv \tau_{1}^{\prime} \rightarrow \tau_{2}^{\prime} \wedge \tau_{1}^{\prime} \sqsubseteq \tau_{1} \wedge \tau_{2} \sqsubseteq \tau_{2}^{\prime}$

$3 \quad \theta \sqsubseteq d^{s} \vec{\tau} \Rightarrow \theta \equiv d^{r} \vec{\sigma} \wedge r \preccurlyeq s \wedge \vec{\sigma} \sqsubseteq \vec{\tau}$ 
$4 \quad d^{s} \vec{\tau} \sqsubseteq \theta \Rightarrow \theta \equiv d^{r} \vec{\sigma} \wedge s \preccurlyeq r \wedge \vec{\tau} \sqsubseteq \vec{\sigma}$

$5 \alpha \sqsubseteq \sigma \Rightarrow \sigma \equiv \alpha$

$6 \quad \sigma \sqsubseteq \alpha \Rightarrow \sigma \equiv \alpha$

Proof. Immediate by analysis of the subtyping rules.

\section{Lemma 3.2 (Generation lemma for typing).}

$1 \quad \Gamma \vdash x: \sigma \Rightarrow(x: \tau) \in \Gamma \wedge \tau \sqsubseteq \sigma$

$2 \Gamma \vdash a b: \sigma \Rightarrow \Gamma \vdash a: \tau \rightarrow \sigma^{\prime} \wedge \Gamma \vdash b: \tau \wedge \sigma^{\prime} \sqsubseteq \sigma$

$3 \quad \Gamma \vdash \lambda x . e: \sigma \Rightarrow \sigma \equiv \tau_{1} \rightarrow \tau_{2} \wedge \Gamma, x: \tau_{1}^{\prime} \vdash e: \tau_{2}^{\prime} \wedge \tau_{1} \sqsubseteq \tau_{1}^{\prime} \wedge \tau_{2}^{\prime} \sqsubseteq \tau_{2}$

$4 \quad \Gamma \vdash c: \sigma \Rightarrow \sigma \equiv \vec{\gamma} \rightarrow \theta \wedge \vec{\gamma} \sqsubseteq \operatorname{lnst}_{c}^{s} \vec{\tau} \wedge d^{\hat{s}} \vec{\tau} \sqsubseteq \theta \wedge c \in \mathrm{C}(d)$

$5 \quad \Gamma \vdash$ case $a$ of $\{\vec{c} \Rightarrow \vec{b}\}: \sigma \Rightarrow \Gamma \vdash a: d^{\widehat{s}} \vec{\tau} \wedge \Gamma \vdash b_{i}:$ Inst $_{c_{i}}^{s} \vec{\tau} \rightarrow \theta \wedge \theta \sqsubseteq \sigma$

$6 \quad \Gamma \vdash$ letrec $f=e: \sigma \Rightarrow \Gamma, f: d^{2} \vec{\tau} \rightarrow \theta \vdash e:\left(d^{2} \vec{\tau} \rightarrow \theta\right)[\imath:=\widehat{\imath}] \wedge\left(d^{2} \vec{\tau} \rightarrow \theta\right)[\imath:=$ $s] \sqsubseteq \sigma$ with $\imath \in \mathcal{V}_{\mathcal{S}}, \imath$ pos $\theta$ and $\imath$ fresh in $\Gamma, \vec{\tau}$

Proof. By inspection on the derivation of the antecedent judgments.

\section{Lemma 3.3.}

$1 \quad$ If $\imath$ pos $\theta$ and $r \preccurlyeq s$, then $\theta[\imath:=r] \sqsubseteq \theta[\imath:=s]$.

2 If $\imath$ neg $\theta$ and $r \preccurlyeq s$, then $\theta[\imath:=s] \sqsubseteq \theta[\imath:=r]$.

3 If $\tau \sqsubseteq \sigma$ and $\alpha$ pos $\theta$, then $\theta[\alpha:=\tau] \sqsubseteq \theta[\alpha:=\sigma]$.

4 If $\tau \sqsubseteq \sigma$ and $\alpha$ neg $\theta$, then $\theta[\alpha:=\sigma] \sqsubseteq \theta[\alpha:=\tau]$.

Proof. Properties 1 and 2 are proved by simultaneous induction on the structure of $\theta$ and similarly for properties 3 and 4 .

\section{Lemma 3.4.}

1 If $\sigma \sqsubseteq \sigma^{\prime}$, then $\sigma[\imath:=s] \sqsubseteq \sigma^{\prime}[\imath:=s]$.

2 If $\sigma \sqsubseteq \sigma^{\prime}$, then $\sigma[\alpha:=\tau] \sqsubseteq \sigma^{\prime}[\alpha:=\tau]$.

Proof. By induction on the structure of $\sigma$.

Lemma 3.5. If $r \preccurlyeq s$ and $\vec{\tau} \sqsubseteq \vec{\sigma}$, then $\operatorname{Inst}_{c}^{r} \vec{\tau} \sqsubseteq \operatorname{Inst}_{c}^{s} \vec{\sigma}$.

Proof. Follows from the previous lemmas.

Lemma 3.6. $\Gamma_{1}, x: \tau, \Gamma_{2}, \Gamma_{3} \vdash a: \sigma \Rightarrow \Gamma_{1}, \Gamma_{2}, x: \tau, \Gamma_{3} \vdash a: \sigma$

Proof. By induction on the derivation of $\Gamma_{1}, x: \tau, \Gamma_{2}, \Gamma_{3} \vdash a: \sigma$.

Lemma 3.7 (Substitution lemma).

If $\Gamma, x: \tau \vdash a: \sigma$ and $\Gamma \vdash b: \tau$, then $\Gamma \vdash a[x:=b]: \sigma$.

Proof. By induction on the derivation of $\Gamma, x: \tau \vdash a: \sigma$.

The following lemma shows the polymorphic nature of stage variables. In fact, in a derivable judgment a stage variable can be replaced throughout by a stage without affecting derivability.

Lemma 3.8. If $\Gamma \vdash a: \sigma$ then $\Gamma[\imath:=s] \vdash a: \sigma[\imath:=s]$. 
Proof. Without loss of generality, one can assume $\imath$ nocc $s$, otherwise one could firstly apply this weaker version of the lemma with $\imath$ being replaced by a new stage variable $\kappa$ (for the set of stage variables is infinite) and use again the weaker version of the lemma with $\kappa$ replaced by $s$.

By induction on the derivation of $\Gamma \vdash a: \sigma$. The only interesting case is when the last rule applied is (rec). (The other cases can be easily proved using the induction hypothesis.) Assume the last step is

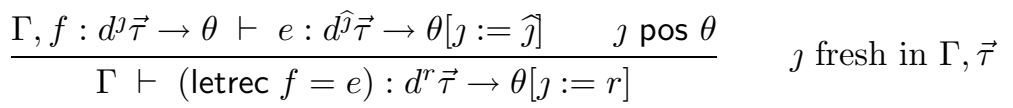

A stage variable $\kappa$ can be chosen such that $\kappa$ is fresh in $\Gamma, \vec{\tau}, \theta, \kappa \neq \imath$ and $\kappa$ nocc $s$. Then, from the induction hypothesis, $\Gamma, f: d^{\kappa} \vec{\tau} \rightarrow \theta[\jmath:=\kappa] \vdash e: d^{\kappa} \vec{\tau} \rightarrow \theta[\jmath:=\widehat{\jmath}][\jmath:=\kappa]$. Therefore $\Gamma, f: d^{\kappa} \vec{\tau} \rightarrow \theta[\jmath:=\kappa] \vdash e: d^{\widehat{\kappa}} \vec{\tau} \rightarrow \theta[\jmath:=\kappa][\kappa:=\widehat{\kappa}]$ and therefore, using again the induction hypothesis,

$$
\begin{array}{ll}
\Gamma[\imath:=s], & f: d^{\kappa} \vec{\tau}[\imath:=s] \rightarrow \theta[\jmath:=\kappa][\imath:=s] \vdash \\
& e: d^{\widehat{\kappa}} \vec{\tau}[\imath:=s] \rightarrow \theta[\jmath:=\kappa][\kappa:=\widehat{\kappa}][\imath:=s]
\end{array}
$$

Since $\kappa$ nocc $s$, the substitutions $[\kappa:=\widehat{\kappa}]$ and $[\imath:=s]$ can be exchanged, obtaining

$$
\begin{aligned}
\Gamma[\imath:=s], \quad & f: d^{\kappa} \vec{\tau}[\imath:=s] \rightarrow \theta[\jmath:=\kappa][\imath:=s] \vdash \\
e & : d^{\widehat{\kappa}} \vec{\tau}[\imath:=s] \rightarrow \theta[\jmath:=\kappa][\imath:=s][\kappa:=\widehat{\kappa}]
\end{aligned}
$$

and, as $\kappa$ is fresh in $\Gamma[\imath:=s]$ and in $\vec{\tau}[\imath:=s]$, one can apply the rule (rec).

Let $u \equiv r[\imath:=s]$. From (1) by (rec),

$$
\Gamma[\imath:=s] \vdash(\text { letrec } f=e): d^{u} \vec{\tau}[\imath:=s] \rightarrow \theta[\jmath:=\kappa][\imath:=s][\kappa:=u]
$$

So, as $\kappa$ nocc $s, \imath$ nocc $s$ and $\kappa$ is $\theta$-fresh, $\Gamma[\imath:=s] \vdash($ letrec $f=e):\left(d^{r} \vec{\tau}\right)[\imath:=s] \rightarrow$ $\theta[\jmath:=u][\imath:=s]$. Hence, $\Gamma[\imath:=s] \vdash($ letrec $f=e):\left(d^{r} \vec{\tau} \rightarrow \theta[\jmath:=r]\right)[\imath:=s]$.

We are now ready to prove that $\lambda$ enjoys the property of subject reduction.

\section{Proposition 3.9 (Subject reduction).}

$$
\Gamma \vdash e_{1}: \sigma \wedge e_{1} \rightarrow \beta \iota \mu, e_{2} \Rightarrow \Gamma \vdash e_{2}: \sigma
$$

Proof. By induction on the derivation of $\Gamma \vdash e_{1}: \sigma$. The interesting cases are when the last rule applied is (app) or (case):

(app)Assume $e_{1} \equiv a b$ and the last step is

$$
\frac{\Gamma \vdash a: \tau_{1} \rightarrow \tau_{2} \quad \Gamma \vdash b: \tau_{1}}{\Gamma \vdash a b: \tau_{2}}
$$

Then one may have the following cases:

$e_{2} \equiv e[x:=b]$, with $a \equiv \lambda x . e$. From the typing derivation for $a$, using Lemma 3.2, follows that $\Gamma, x: \tau_{1}^{\prime} \vdash e: \tau_{2}^{\prime}, \tau_{1} \sqsubseteq \tau_{1}^{\prime}$ and $\tau_{2}^{\prime} \sqsubseteq \tau_{2}$, and from the typing derivation for $b$, by (sub) one derives $\Gamma \vdash b: \tau_{1}^{\prime}$. Thus, by Lemma 3.7, $\Gamma \vdash e[x:=b]: \tau_{2}^{\prime}$ and finally by the rule (sub), $\Gamma \vdash e[x:=b]: \tau_{2}$. 
$e_{2} \equiv(e[f:=($ letrec $f=e)])(c \vec{a})$, with $a \equiv($ letrec $f=e)$ and $b \equiv(c \vec{a})$. Applying Lemma 3.2 to the typing derivation for $a$ one has:

$$
\begin{aligned}
\Gamma, f: d^{2} \vec{\tau} \rightarrow \theta \vdash e:\left(d^{2} \vec{\tau} \rightarrow \theta\right)[\imath:=\widehat{\imath}] \\
\left(d^{2} \vec{\tau} \rightarrow \theta\right)[\imath:=s] \sqsubseteq \tau_{1} \rightarrow \tau_{2} \\
\imath \in \mathcal{V}_{\mathcal{S}} \wedge \imath \operatorname{pos} \theta \wedge \imath \text { fresh in } \Gamma, \vec{\tau}
\end{aligned}
$$

From (3) by Lemma 3.1, $\tau_{1} \sqsubseteq d^{s} \vec{\tau} \wedge \theta[\imath:=s] \sqsubseteq \tau_{2}$ and thus, using again Lemma 3.1 ,

$$
\tau_{1} \equiv d^{p} \overrightarrow{\tau^{\prime}} \wedge p \preccurlyeq s \quad \wedge \quad \overrightarrow{\tau^{\prime}} \sqsubseteq \vec{\tau}
$$

From (2) and (4), by (rec), $\Gamma \vdash$ (letrec $f=e):\left(d^{2} \vec{\tau} \rightarrow \theta\right)[\imath:=q]$ holds for an arbitary stage $q$. Therefore, choosing $q \equiv \imath$ and taking into account (2), by Lemma 3.7 one can derive

$$
\Gamma \vdash e[f:=(\text { letrec } f=e)]:\left(d^{2} \vec{\tau} \rightarrow \theta\right)[\imath:=\widehat{\imath}]
$$

Thus we have $\Gamma \vdash(c \vec{a}): d^{p} \overrightarrow{\tau^{\prime}}$ and so, by lemmas 3.1 and 3.2, one of two possibilities for $p$ must arise: $p \equiv \jmath^{n}$ with $n \geq 1$ or $p \equiv \infty^{m}$ with $m \geq 0$, where for a stage $s$ and for $k \in \mathbb{N}, s^{k}$ means $s$ hatted $k$ times.

- Case $p \equiv \jmath^{n}$ with $n \geq 1$ then, using Lemma 3.8 on (5) with substitution $\left[\imath:=\jmath^{(n-1)}\right]$, and since $\imath$ is fresh w.r.t. $\Gamma$ and $\vec{\tau}$,

$$
\Gamma \vdash e[f:=(\text { letrec } f=e)]: d^{\eta^{n}} \vec{\tau} \rightarrow \theta\left[\imath:=\jmath^{n}\right]
$$

Thus, since $\Gamma \vdash(c \vec{a}): \tau_{1}$ and $\tau_{1} \equiv d^{p} \overrightarrow{\tau^{\prime}}$, by (sub) and (app), follows $\Gamma \vdash$ $e[f:=($ letrec $f=e)](c \vec{a}): \theta\left[\imath:=\jmath^{n}\right]$. One has $\jmath^{n} \preccurlyeq s$ and $\imath$ pos $\theta$ so, by Lemma $3.3, \theta\left[\imath:=\jmath^{n}\right] \sqsubseteq \theta[\imath:=s]$ and the proof of this case is concluded using the rule (sub).

— Case $p \equiv \infty^{m}$ with $m \geq 0$ then, observing that by (sub) $\Gamma \vdash(c \vec{a}): d^{\infty^{(m+1)}} \overrightarrow{\tau^{\prime}}$, and the proof could now be completed arguing as in the previous case.

The remaining cases, where $e_{2} \equiv a^{\prime} b$ with $a \rightarrow_{\beta \iota \mu} a^{\prime}$, or $e_{2} \equiv a b^{\prime}$ with $b \rightarrow_{\beta \iota \mu} b^{\prime}$, follow by routine induction.

(case)Assume $e_{1} \equiv$ case $a$ of $\left\{c_{1} \Rightarrow b_{1}|\ldots| c_{n} \Rightarrow b_{n}\right\}$ and the last step is

$$
\frac{\Gamma \vdash a: d^{s} \vec{\tau} \quad \Gamma \vdash b_{i}: \text { Inst }_{c_{i}}^{s} \vec{\tau} \rightarrow \theta \quad(1 \leq i \leq n)}{\Gamma \vdash \text { case } a \text { of }\left\{c_{1} \Rightarrow b_{1}|\ldots| c_{n} \Rightarrow b_{n}\right\}: \theta}
$$

Then one may have:

$e_{2} \equiv b_{i} a_{1} \ldots a_{\operatorname{ar}\left(c_{i}\right)}$, with $a \equiv c_{i} a_{1} \ldots a_{\operatorname{ar}\left(c_{i}\right)}$. From $\Gamma \vdash c_{i} a_{1} \ldots a_{\operatorname{ar}\left(c_{i}\right)}: d^{\widehat{s}} \vec{\tau}$, by Lemma 3.2 , it follows that

$$
\Gamma \vdash c_{i}: \vec{\gamma} \rightarrow \sigma \wedge \sigma \sqsubseteq d^{\widehat{s}} \vec{\tau}
$$

and also for $1 \leq j \leq \operatorname{ar}\left(c_{i}\right)$

$$
\Gamma \vdash a_{j}: \gamma_{j} \wedge \gamma_{j} \sqsubseteq \operatorname{Inst}_{c_{i}}^{r} \vec{\psi}[j] \wedge d^{\widehat{r}} \vec{\psi} \sqsubseteq \sigma
$$

So, $d^{\widehat{r}} \vec{\psi} \sqsubseteq d^{\widehat{s}} \vec{\tau}$ and therefore, by lemmas 3.1 and 2.14, $r \preccurlyeq s$ and $\vec{\psi} \sqsubseteq \vec{\tau}$. Using 
Lemma 3.5 and the (sub) rule, one has $\Gamma \vdash a_{j}: \operatorname{Inst}_{c_{i}}^{s} \vec{\tau}[j]$ for $1 \leq j \leq \operatorname{ar}\left(c_{i}\right)$, which can be combined with the typing derivation of $b_{i}$, by means of the rule (app), to conclude the proof of this case.

The remaining cases, $e_{2} \equiv$ case $a^{\prime}$ of $\{\vec{c} \Rightarrow \vec{b}\}$ with $a \rightarrow_{\beta \iota \mu} a^{\prime}$, and $e_{2} \equiv$ case $a^{\prime}$ of $\left\{c_{1} \Rightarrow\right.$ $\left.b_{1}|\ldots| c_{i} \Rightarrow b_{i}^{\prime}|\ldots| c_{n} \Rightarrow b_{n}\right\}$ with $b_{i} \rightarrow_{\beta \iota \mu} b_{i}^{\prime}$, follow by routine induction.

\subsection{Strong normalization}

As usual, we say that a term $e$ is strongly normalizing with respect to $\rightarrow_{\beta \iota \mu}$, if all $\beta \iota \mu-$ reduction sequences starting with $e$ terminate. Let $\mathrm{SN}$ denote the set of terms that are strongly normalizing with respect to $\rightarrow \beta \iota \mu$.

To prove that every typable term is in SN, we use the method of saturated sets. This is a standard technique, see, e.g., (Luo 1994). The idea is to provide the system with a semantics in which terms are interpreted as terms and type expressions as sets of terms known by construction only to contain strongly normalizing terms and always be non-empty (saturated sets). The strong normalizability of all typable terms then follows immediately as soon it is proved that the system is sound with respect to it.

3.2.1. Saturated sets and interpretation domains We start by defining the notions of base terms and key reduction (aka. weak head reduction).

Definition 3.10 (Base terms). The set Base of base terms is defined inductively as follows:

$-\mathcal{V}_{\mathcal{E}} \subseteq$ Base.

- If $b \in$ Base and $e \in \mathrm{SN}$ then $b e \in$ Base.

- If $b \in$ Base and $e_{1}, \ldots, e_{n} \in \mathrm{SN}$, then case $b$ of $\left\{c_{1} \Rightarrow e_{1}|\ldots| c_{n} \Rightarrow e_{n}\right\} \in$ Base.

- If $b \in$ Base and $e \in \mathrm{SN}$ then (letrec $f=e$ ) $b \in$ Base.

Every base term is strongly normalizing.

Lemma 3.11. Base $\subseteq \mathrm{SN}$

Definition 3.12 (Key reduction). The relation of key reduction between terms is defined inductively as follows:

- If $e$ is a $\beta \iota \mu$-redex and $e^{\prime}$ is the contractum, then $e \rightarrow_{k} e^{\prime}$.

- If $a \rightarrow_{k} a^{\prime}$, then $a e \rightarrow_{k} a^{\prime} e$,

- If $a \rightarrow_{k} a^{\prime}$, then case $a$ of $\left\{c_{1} \Rightarrow e_{1}|\ldots| c_{n} \Rightarrow e_{n}\right\} \rightarrow_{k}$ case $a^{\prime}$ of $\left\{c_{1} \Rightarrow e_{1}|\ldots|\right.$ $\left.c_{n} \Rightarrow e_{n}\right\}$.

- If $a \rightarrow_{k} a^{\prime}$, then (letrec $\left.f=e\right) a \rightarrow_{k}$ (letrec $\left.f=e\right) a^{\prime}$.

Key reduction commutes with reduction in the following sense.

Lemma 3.13. If $a \rightarrow_{k} b$ and $a \rightarrow a^{\prime} \neq b$, then $a^{\prime} \rightarrow_{k} b^{\prime}$ and $b \rightarrow b^{\prime}$ for some $b^{\prime}$.

The following two lemmas provide sufficient conditions for an expression to be strongly normalizing. 


\section{Lemma 3.14.}

1 If $a \in \mathrm{SN}, a \rightarrow_{k} b$ and $b e \in \mathrm{SN}$, then $a e \in \mathrm{SN}$.

2 If $a \in \mathrm{SN}, a \rightarrow_{k} b$ and case $b$ of $\left\{c_{1} \Rightarrow e_{1}|\ldots| c_{n} \Rightarrow e_{n}\right\} \in \mathrm{SN}$, then case $a$ of $\left\{c_{1} \Rightarrow\right.$ $\left.e_{1}|\ldots| c_{n} \Rightarrow e_{n}\right\} \in \mathrm{SN}$.

3 If $a \in \mathrm{SN}, a \rightarrow_{k} b$ and (letrec $f=e$ ) $b \in \mathrm{SN}$, then (letrec $f=e$ ) $a \in \mathrm{SN}$.

Proof. We prove (1). Suppose $a \in \mathrm{SN}, a \rightarrow_{k} b$ and $b e \in \mathrm{SN}$. First note that $e \in \mathrm{SN}$ as $b e \in \mathrm{SN}$. The proof of $a e \in \mathrm{SN}$ is by simultaneous induction on " $a \in \mathrm{SN}$ " and " $e \in \mathrm{SN}$ ". We have to prove that $c \in \mathrm{SN}$ for any one $c$ such that $a e \rightarrow c$. As $a$ can be neither a lambda-abstraction nor a letrec, there are two cases: either $c=a^{\prime} e$ and $a \rightarrow a^{\prime}$ or $c=a e^{\prime}$ and $e \rightarrow e^{\prime}$.

- Suppose $c=a^{\prime} e$ and $a \rightarrow a^{\prime}$. If $a^{\prime}=b$, then $c=a^{\prime} e \in \mathrm{SN}$, since $b e \in \mathrm{SN}$. Otherwise, by Lemma 3.13, there is $b^{\prime}$ such that $a^{\prime} \rightarrow_{k} b^{\prime}$ and $b \rightarrow b^{\prime}$. We have $a^{\prime} \in \mathrm{SN}$ (as $a \in \mathrm{SN}$ ), $a^{\prime} \rightarrow_{k} b^{\prime}$ and $b^{\prime} e \in \mathrm{SN}$ (as $b e \in \mathrm{SN}$ ). By the induction hypothesis, $c=a^{\prime} e \in \mathrm{SN}$.

- Suppose $c=a e^{\prime}$ and $e \rightarrow e^{\prime}$. We have $a \in \mathrm{SN}, a \rightarrow_{k} b, b e^{\prime} \in \mathrm{SN}$ (as $b e \in \mathrm{SN}$ ). By the induction hypothesis, $c=a e^{\prime} \in \mathrm{SN}$.

\section{Lemma 3.15.}

1 If $a, e, a[x:=e] \in \mathrm{SN}$, then $(\lambda x . a) e \in \mathrm{SN}$.

2 If $\vec{a}, e_{1}, \ldots, e_{n}, e_{i} \vec{a} \in \mathrm{SN}$, then case $\left(c_{i} \vec{a}\right)$ of $\left\{c_{1} \Rightarrow e_{1}|\ldots| c_{n} \Rightarrow e_{n}\right\} \in \mathrm{SN}$.

3 If $\vec{a}, e, e[f:=($ letrec $f=e)](c \vec{a}) \in \mathrm{SN}$, then (letrec $f=e)(c \vec{a}) \in \mathrm{SN}$.

Next we define saturated sets and state some of their closure properties. Saturated sets are sets of strongly normalizing terms containing the base terms and closed with respect to key expansion.

\section{Definition 3.16 (Saturated sets).}

1 A set $X \subseteq \mathcal{E}$ is said to be a saturated set, if

$-X \subseteq \mathrm{SN}$,

- Base $\subseteq X$,

- if $a \in \mathrm{SN}$ and $a \rightarrow_{k} a^{\prime}$ for some $a^{\prime} \in X$, then $a \in X$.

The set of all saturated sets is denoted by SAT.

2 For any $X \subseteq \mathcal{E}$, let $\ulcorner X\urcorner=\left\{a \in \mathrm{SN} \mid \exists b \in\right.$ Base $\left.\cup X . a \rightarrow_{k} b\right\}$.

The following lemma establishes some basic properties of the closure operator $\ulcorner$.$\urcorner .$

\section{Lemma 3.17.}

1 If $X \subseteq \mathrm{SN}$, then $\ulcorner X\urcorner$ is a saturated set, in fact, the smallest saturated set containing $X$.

$2\left\ulcorner X_{1} \cup \ldots \cup X_{n}\right\urcorner=\left\ulcorner X_{1}\right\urcorner \cup \ldots \cup\left\ulcorner X_{n}\right\urcorner$.

3 If $X_{i}$ is a saturated set for any $i \in I$, then $\bigcup i \in I . X_{i}$ is a saturated set. (We say that $\left.\bigcup i \in \emptyset . X_{i}=\ulcorner\emptyset\urcorner.\right)$ 
On saturated sets, we can define a function-space forming operation. This is needed for the interpretation of function-space types.

Definition 3.18. For any $X, Y \subseteq \mathcal{E}$, let $X \rightarrow Y=\{a \in \mathcal{E} \mid \forall e \in X$. ae $\in Y\}$.

Lemma 3.19. If $X$ and $Y$ are saturated sets, then so is $X \rightarrow Y$.

Proof. Suppose $X$ and $Y$ are saturated. Clearly any $a \in X \rightarrow Y$ is strongly normalizing: as $X$ is non-empty, we can pick some $e \in X$ and then $a \in \mathrm{SN}$ because $a e \in Y \subseteq \mathrm{SN}$. Let us check that $X \rightarrow Y$ satisfies the conditions of saturatedness.

- Suppose $b \in$ Base and consider any $e \in X$. As $e \in \mathrm{SN}$, we have that $b e \in$ Base $\subseteq Y$. Hence $b \in X \rightarrow Y$.

- Suppose $a \in \mathrm{SN}, a \rightarrow_{k} a^{\prime}$ and $a^{\prime} \in X \rightarrow Y$. We have to show that $a \in X \rightarrow Y$, i.e., that, for any $e \in X$, ae $\in Y$. Consider any $e \in X$. We have $a e \rightarrow_{k} a^{\prime} e$ and $a^{\prime} e \in Y \subseteq \mathrm{SN}$, hence Lemma 3.14 applies and $a e \in \mathrm{SN}$. Since $Y$ is saturated, we get $a e \in Y$.

3.2.2. Type and term interpretation In what now follows, we define a semantics of the language of stages, types, and terms and show that the rules of stage comparison, subtyping and typing are sound with respect to that semantics. Types will be interpreted as saturated sets of terms, terms will be interpreted as terms.

We start with the definitions of valuations and interpretation for stages and types. Stages will be interpreted as ordinals below $\Omega$, the first uncountable ordinal, types as saturated sets of terms. Inductive types are interpreted as limits of a monotone approximation process from below. As the universe, SN, is countable, the approximation process is guaranteed to converge before $\Omega$.

\section{Definition 3.20 (Stage valuation).}

1 A stage valuation is a map $\pi: \mathcal{V}_{\mathcal{S}} \rightarrow \Omega+1$.

2 For every stage valuation $\pi, \imath \in \mathcal{V}_{\mathcal{S}}$, and $x \in \Omega+1$, the stage valuation $\pi(\imath:=x)$ is defined as follows:

$$
\pi(\imath:=x)\left(\iota^{\prime}\right)= \begin{cases}x & \text { if } \iota^{\prime} \equiv \iota \\ \pi\left(\iota^{\prime}\right) & \text { if } \iota^{\prime} \neq \equiv \iota\end{cases}
$$

Definition 3.21 (Interpretation of stages). Let $\pi$ be a type valuation. The corresponding stage interpretation function $\llbracket \cdot \rrbracket_{\pi}: \mathcal{S} \rightarrow \Omega+1$ is defined as follows:

$$
\begin{aligned}
\llbracket \imath \rrbracket_{\pi} & =\pi(\imath) \text { if } \imath \in \mathcal{V}_{\mathcal{S}} \\
\llbracket \infty \rrbracket_{\pi} & =\Omega \\
\llbracket \hat{s} \rrbracket_{\pi} & = \begin{cases}\llbracket s \rrbracket_{\pi}+1 & \text { if } \llbracket s \rrbracket_{\pi}<\Omega \\
\llbracket s \rrbracket_{\pi} & \text { if } \llbracket s \rrbracket_{\pi}=\Omega\end{cases}
\end{aligned}
$$

Definition 3.22 (Type valuation).

1 A type valuation is a map $\xi: \mathcal{V}_{\mathcal{T}} \rightarrow$ SAT. 
2 For every type valuation $\xi, \alpha \in \mathcal{V}_{\mathcal{T}}$, and $X \in \mathrm{SAT}$, the type valuation $\xi(\alpha:=X)$ is defined as follows:

$$
\xi(\alpha:=X)\left(\alpha^{\prime}\right)= \begin{cases}X & \text { if } \alpha^{\prime} \equiv \alpha \\ \xi\left(\alpha^{\prime}\right) & \text { if } \alpha^{\prime} \neq \alpha\end{cases}
$$

Definition 3.23 (Interpretation of types). Let $\pi$ be a stage valuation and $\xi$ a type valuation. The corresponding type interpretation function $\llbracket \cdot \rrbracket_{\pi, \xi}: \mathcal{T} \rightarrow$ SAT is defined by induction on heights (because of the stratification on datatype identifiers, every type has finite height):

$$
\begin{aligned}
\llbracket \alpha \rrbracket_{\pi, \xi} & =\xi(\alpha) \text { if } \alpha \in \mathcal{V}_{\mathcal{T}} \\
\llbracket \tau \rightarrow \sigma \rrbracket_{\pi, \xi} & =\llbracket \tau \rrbracket_{\pi, \xi} \rightarrow \llbracket \sigma \rrbracket_{\pi, \xi} \\
\llbracket d^{s} \vec{\tau} \rrbracket_{\pi, \xi} & =D\left(\llbracket \vec{\tau} \rrbracket_{\pi, \xi}, \llbracket s \rrbracket_{\pi}\right)
\end{aligned}
$$

where $D(\vec{X}, x)$ is defined by induction on $x$ by

$$
\begin{aligned}
D(\vec{X}, 0) & =\ulcorner\emptyset\urcorner \\
D(\vec{X}, y+1)= & \left\ulcorner c_{1} \llbracket \overrightarrow{\sigma_{1}} \rrbracket_{\pi, \xi(\delta:=D(\vec{X}, y), \vec{\alpha}:=\vec{X})} \cup \ldots \cup c_{n} \llbracket \overrightarrow{\sigma_{n}} \rrbracket_{\pi, \xi(\delta:=D(\vec{X}, y), \vec{\alpha}:=\vec{X})}\right\urcorner \\
& \text { where } \mathrm{D}\left(c_{i}\right)=\left(\delta, \vec{\alpha}, \overrightarrow{\sigma_{i}}\right) \\
D(\vec{X}, x)= & \bigcup y<x . D(\vec{X}, y) \text { if } x \text { is a limit ordinal }
\end{aligned}
$$

Lemma 3.24 (Substitution lemma for the interpretation of types).

$1 \llbracket\left[\sigma[\iota:=s] \rrbracket_{\pi, \xi}=\llbracket \sigma \rrbracket_{\pi\left(\iota:=\llbracket s \rrbracket_{\pi}\right), \xi}\right.$

$2 \quad \llbracket \sigma[\alpha:=\tau] \rrbracket_{\pi, \xi}=\llbracket \sigma \rrbracket_{\pi, \xi\left(\alpha:=\llbracket \tau \rrbracket_{\pi, \xi}\right)}$

The following lemma states that the sequence of approximates of any datatype is non-decreasing with respect to set inclusion and converges before $\Omega$.

\section{Lemma 3.25.}

1 If $X \subseteq X^{\prime}$ and $\alpha$ pos $\sigma$, then $\llbracket \sigma \rrbracket_{\pi, \xi(\alpha:=X)} \subseteq \llbracket \sigma \rrbracket_{\pi, \xi\left(\alpha:=X^{\prime}\right)}$.

If $X \subseteq X^{\prime}$ and $\alpha$ neg $\sigma$, then $\llbracket \sigma \rrbracket_{\pi, \xi\left(\alpha:=X^{\prime}\right)} \subseteq \llbracket \sigma \rrbracket_{\pi, \xi(\alpha:=X)}$.

2 If $x \leq x^{\prime}$, then $D(\vec{X}, x) \subseteq D\left(\vec{X}, x^{\prime}\right)$.

$3 \quad D(\vec{X}, \Omega+1)=D(\vec{X}, \Omega)$.

Proof.

1 By mutual induction on the height of $\sigma$.

2 From (1), by induction on $x$.

3 From (2), using the fact that $\mathcal{E}$ is countable. The iteration process has to converge before $\Omega$ : the opposite would imply that $\mathcal{E}$ is uncountable, as $\Omega$ is uncountable.

\section{Lemma 3.26.}

$1 \llbracket \llbracket d^{\widehat{s}} \vec{\tau} \rrbracket_{\pi, \xi}=\left\ulcorner c_{1} \llbracket \operatorname{lnst}_{c_{1}}^{s} \vec{\tau} \rrbracket_{\pi, \xi} \cup \ldots \cup c_{n} \llbracket \operatorname{lnst}_{c_{n}}^{s} \vec{\tau} \rrbracket_{\pi, \xi}\right\urcorner$

$2 \llbracket \llbracket d^{\infty} \vec{\tau} \rrbracket_{\pi, \xi}=\left\ulcorner c_{1} \llbracket \operatorname{lnst}_{c_{1}}^{\infty} \vec{\tau} \rrbracket_{\pi, \xi} \cup \ldots \cup c_{n} \llbracket \operatorname{lnst}_{c_{n}}^{\infty} \vec{\tau} \rrbracket_{\pi, \xi}\right\urcorner$

Next we define valuations and interpretation for terms. 


\section{Definition 3.27 (Term valuation).}

1 A term valuation is a map $\rho: \mathcal{V}_{\mathcal{E}} \rightarrow \mathcal{E}$.

2 For every term valuation $\rho, e \in \mathcal{E}$ and $x \in \mathcal{V}_{\mathcal{E}}$, the term valuation $\rho(x:=e)$ is defined as follows:

$$
\rho(x:=e)(z)= \begin{cases}e & \text { if } z \equiv x \\ \rho(z) & \text { if } z \not \equiv x\end{cases}
$$

Definition 3.28 (Interpretation of terms). For any term valuation $\rho$, the map $(\text { (I.) })_{\rho}: \mathcal{E} \rightarrow \mathcal{E}$ is defined inductively as follows:

$$
\begin{aligned}
\left([x)_{\rho}\right. & =\rho(x) \\
\left([\lambda x . e)_{\rho}\right. & =\lambda x \cdot\left([e)_{\rho(x:=x)}\right. \\
\left(\left[e e^{\prime}\right)_{\rho}\right. & =(l e)_{\rho}\left(\left[e^{\prime}\right]\right)_{\rho} \\
\left(\left[c_{k}\right)_{\rho}\right. & =c_{k} \\
\text { (Icase } \left.e \text { of }\left\{c_{1} \Rightarrow e_{1}|\ldots| c_{n} \Rightarrow e_{n}\right\}\right)_{\rho} & =\text { case }([e])_{\rho} \text { of }\left\{c_{1} \Rightarrow\left(\left[e_{1}\right]_{\rho}|\ldots| c_{n} \Rightarrow\left(\left[e_{n}\right]\right)_{\rho}\right\}\right. \\
(\text { letrec } f=e]_{\rho} & =\text { letrec } f=([e])_{\rho(f:=f)}
\end{aligned}
$$

Remark 3.29. In the clauses for lambda-abstraction and letrec, a form of variable convention is relied upon: namely, $x$ resp. $f$ is assumed not to appear as a free variable in any of the terms $\rho(y)$ where $y$ is free in $e$. Alternatively, without the convention, some variable renaming may be necessary: in the case of lambda-abstraction, one would set

$$
([\lambda x . e])_{\rho}=\lambda x^{\prime} \cdot\left([e)_{\rho\left(x:=x^{\prime}\right)}\right.
$$

where $x^{\prime}$ is some variable free in no $\rho(y)$ where $y$ is free in $e$.

\section{Lemma 3.30 (Substitution lemma for the interpretation of terms).}

- $\left(\left[e\left[x:=e^{\prime}\right]\right)_{\rho}=([e])_{\rho\left(x:=\left(\left[e^{\prime}\right)_{\rho}\right)\right.}\right.$.

- $(e]_{\rho}=e[\vec{y}:=\rho(\vec{y})]$ where $\vec{y}$ are the free variables of $e$.

The notion of satisfaction and validity are defined as usual: satisfaction of subtyping is set inclusion, and satisfaction of typing is set membership.

\section{Definition 3.31 (Satisfaction, validity).}

1 A stage valuation $\pi$ satisfies a stage comparison judgment $s \preccurlyeq s^{\prime}$, if $\llbracket s \rrbracket_{\pi} \leq \llbracket s^{\prime} \rrbracket_{\pi}$. A stage comparison judgment $s \preccurlyeq s^{\prime}$ is valid, if every stage valuation satisfies it.

2 A stage valuation $\pi$ and a type valuation $\xi$ satisfy a subtyping judgment $\sigma \sqsubseteq \sigma^{\prime}$, if $\llbracket \sigma \rrbracket_{\pi, \xi} \subseteq \llbracket \sigma^{\prime} \rrbracket_{\pi, \xi}$. A subtyping judgment $\sigma \sqsubseteq \sigma^{\prime}$ is valid, if every pair of stage and type valuations satisfies it.

3 A valuation is a triple $(\pi, \xi, \rho)$, where $\pi$ is a stage valuation, $\xi$ is a type valuation and $\rho$ is a term valuation.

4 Let $(\pi, \xi, \rho)$ be a valuation.

(a) $(\pi, \xi, \rho)$ satisfies a context $\Gamma$, written $(\pi, \xi, \rho) \models \Gamma$, if $\rho(x) \in \llbracket \tau \rrbracket_{\pi, \xi}$ for each $(x: \tau) \in \Gamma$.

(b) $(\pi, \xi, \rho)$ satisfies a typing judgment $\Gamma \vdash e: \sigma$, if

$$
(\pi, \xi, \rho) \models \Gamma \quad \Rightarrow \quad(e)_{\rho} \in \llbracket \sigma \rrbracket_{\pi, \xi}
$$


5 A typing judgment $\Gamma \vdash e: \sigma$ is valid, written $\Gamma \models e: \sigma$, if every valuation satisfies it.

3.2.3. Soundness wrt. the semantics Next we prove that the rules of $\lambda \uparrow$ for stage comparison, subtyping, and typing are sound wrt. the semantics just defined. The strong normalization theorem follows as a corollary from the typing soundness.

\section{Proposition 3.32 (Stage comparison soundness).}

$$
s \preccurlyeq s^{\prime} \text { derivable } \Rightarrow s \preccurlyeq s^{\prime} \text { valid }
$$

Proof. By induction on the derivation of $s \preccurlyeq s^{\prime}$.

\section{Proposition 3.33 (Subtyping soundness).}

$$
\sigma \sqsubseteq \sigma^{\prime} \text { derivable } \Rightarrow \sigma \sqsubseteq \sigma^{\prime} \text { valid }
$$

Proof. By induction on the derivation of $\sigma \sqsubseteq \sigma^{\prime}$.

Lemma 3.34. Let $\xi$ be a type valuation. Then

1 If $\imath$ pos $\sigma$ and $x \leq x^{\prime}$, then $\llbracket \sigma \rrbracket_{\pi(\imath:=x), \xi} \subseteq \llbracket \sigma \rrbracket_{\pi\left(\imath:=x^{\prime}\right), \xi}$.

2 If $\imath$ neg $\sigma$ and $x \leq x^{\prime}$, then $\llbracket \sigma \rrbracket_{\pi(\imath:=x), \xi} \supseteq \llbracket \sigma \rrbracket_{\pi\left(\imath:=x^{\prime}\right), \xi}$.

Proof. By simultaneous induction on the structure of $\sigma$.

\section{Proposition 3.35 (Typing soundness).}

$$
\Gamma \vdash e: \sigma \text { derivable } \Rightarrow \Gamma \models e: \sigma
$$

Proof. By induction on the derivation of $\Gamma \vdash e: \sigma$.

(var)Assume the last (and the only) step is

$$
\overline{\Gamma \vdash x: \tau} \text { and }(x: \tau) \in \Gamma
$$

Suppose $(\pi, \xi, \rho) \models \Gamma$. We have to show that $\left([x)_{\rho} \in \llbracket \tau \rrbracket_{\pi, \xi}\right.$. This is true, as $(x: \tau) \in \Gamma$.

(abs) Assume the last step is

$$
\frac{\Gamma, x: \tau \vdash e: \sigma}{\Gamma \vdash \lambda x . e: \tau \rightarrow \sigma}
$$

Suppose $(\pi, \xi, \rho) \models \Gamma$. We have to show that $\left([\lambda x . e]_{\rho} \in \llbracket \tau \rightarrow \sigma \rrbracket_{\pi, \xi}\right.$. Since $\llbracket \tau \rightarrow$ $\sigma \rrbracket_{\pi, \xi}=\llbracket \tau \rrbracket_{\pi, \xi} \rightarrow \llbracket \sigma \rrbracket_{\pi, \xi}$ and $\left([\lambda x . e)_{\rho}=\lambda x\right.$. $\left([e)_{\rho_{0}}\right.$, where $\rho_{0}=\rho(x:=x)$, this amounts to showing that $\left(\lambda x\right.$. $\left([e)_{\rho_{0}}\right) a \in \llbracket \sigma \rrbracket_{\pi, \xi}$ for any $a \in \llbracket \tau \rrbracket_{\pi, \xi}$.

Observe first that, since $\left(\pi, \xi, \rho_{0}\right) \models \Gamma$ and $\rho_{0}(x)=x \in \mathcal{V}_{\mathcal{E}} \subseteq \llbracket \tau \rrbracket_{\pi, \xi}$, the induction hypothesis tells us that $\left([e]_{\rho_{0}} \in \llbracket \sigma \rrbracket_{\pi, \xi} \subseteq \mathrm{SN}\right.$.

Suppose now $a \in \llbracket \tau \rrbracket_{\pi, \xi} \subseteq$ SN and let $\rho^{\prime}=\rho(x:=a)$. Since $\left(\pi, \xi, \rho^{\prime}\right) \models \Gamma$ and $\rho^{\prime}(x)=a \in \llbracket \tau \rrbracket_{\pi, \xi}$, by the induction hypothesis, we get that $\left([e)_{\rho^{\prime}} \in \llbracket \sigma \rrbracket_{\pi, \xi} \subseteq \mathrm{SN}\right.$. Write $\vec{y}$ for the free variables of $e$, then $\left(\lambda x .\left([e)_{\rho_{0}}\right) a \rightarrow_{k}(e]_{\rho_{0}}[x:=a]=e[\vec{y}:=\right.$ $\left.\rho_{0}(\vec{y})\right][x:=a]=e\left[\vec{y}:=\rho^{\prime}(\vec{y})\right]=\left([e] \rho_{\rho^{\prime}}\right.$ (by the variable convention, Remark 3.29, $x$ does not occur free in $\left.\rho_{0}(\vec{y})\right)$. By Lemma $3.15,\left(\lambda x .\left([e)_{\rho_{0}}\right) a \in \mathrm{SN}\right.$. As $\llbracket \sigma \rrbracket_{\pi, \xi}$ is a 
saturated set, we get that $\left(\lambda x\right.$. $\left([e)_{\rho_{0}}\right) a \in \llbracket \sigma \rrbracket_{\pi, \xi}$.

(app)Assume the last step is

$$
\frac{\Gamma \vdash e: \tau \rightarrow \sigma \quad \Gamma \vdash e^{\prime}: \tau}{\Gamma \vdash e e^{\prime}: \sigma}
$$

Suppose $(\pi, \xi, \rho) \models \Gamma$. We have to show that $\left(\left[e e^{\prime}\right)_{\rho} \in \llbracket \sigma \rrbracket_{\pi, \xi}\right.$. As $\left(\left[e e^{\prime}\right]_{\rho}=\left([e)_{\rho}\left(\left[e^{\prime}\right]_{\rho}\right.\right.\right.$, this amounts to showing that $\left([e)_{\rho}\left(\left[e^{\prime}\right)_{\rho} \in \llbracket \sigma \rrbracket_{\pi, \xi}\right.\right.$.

As $(\pi, \xi, \rho) \models \Gamma$, the induction hypothesis gives that $\left([e)_{\rho} \in \llbracket \tau \rightarrow \sigma \rrbracket_{\pi, \xi}=\llbracket \tau \rrbracket_{\pi, \xi} \rightarrow\right.$ $\llbracket \sigma \rrbracket_{\pi, \xi}$ and $\left(\left[e^{\prime}\right)_{\rho} \in \llbracket \tau \rrbracket_{\pi, \xi}\right.$. Thus $\left([e]_{\rho}\left(\left[e^{\prime}\right)_{\rho} \in \llbracket \sigma \rrbracket_{\pi, \xi}\right.\right.$.

(cons)Assume the last (and the only) step is

$$
\overline{\Gamma \vdash c_{k}: \operatorname{lnst}_{c_{k}}^{s} \vec{\tau} \rightarrow d^{\widehat{s}} \vec{\tau}} \text { and } k \in 1 . . n
$$

Suppose $(\pi, \xi, \rho) \models \Gamma$. We have to show that $\left(\left[c_{k}\right)_{\rho} \in \llbracket \operatorname{lnst}_{c_{k}}^{s} \vec{\tau} \rightarrow d^{\hat{s}} \vec{\tau} \rrbracket_{\pi, \xi}=\llbracket \operatorname{lnst}_{c_{k}}^{s} \vec{\tau} \rrbracket_{\pi, \xi} \rightarrow\right.$ $\llbracket d^{\hat{s}} \vec{\tau} \rrbracket_{\pi, \xi}$. As $\left(\left[c_{k} \rrbracket_{\rho}=c_{k}\right.\right.$, this amounts to showing that $c_{k} \vec{a} \in \llbracket d^{\hat{s}} \vec{\tau} \rrbracket_{\pi, \xi}$ for any $\vec{a} \in \llbracket \operatorname{lnst}_{c_{k}}^{s} \vec{\tau} \rrbracket_{\pi, \xi}$. But that holds trivially, since $\llbracket d^{\widehat{s}} \vec{\tau} \rrbracket_{\pi, \xi}=\left\ulcorner c_{1} \llbracket \operatorname{Inst}_{c_{1}}^{s} \vec{\tau} \rrbracket_{\pi, \xi} \cup \ldots \cup\right.$ $\left.c_{n} \llbracket \operatorname{lnst}_{c_{n}}^{s} \vec{\tau} \rrbracket_{\pi, \xi}\right\urcorner$.

(case)Assume the last step is

$$
\frac{\Gamma \vdash e: d^{\widehat{s}} \vec{\tau} \quad \Gamma \vdash e_{1}: \text { Inst }_{c_{1}}^{s} \vec{\tau} \rightarrow \theta \quad \ldots \quad \Gamma \vdash e_{n}: \text { Inst }_{c_{n}}^{s} \vec{\tau} \rightarrow \theta}{\Gamma \vdash \text { case } e \text { of }\left\{c_{1} \Rightarrow e_{1}|\ldots| c_{n} \Rightarrow e_{n}\right\}: \theta}
$$

Suppose $(\pi, \xi, \rho) \models \Gamma$. We have to show that ([case $e$ of $\left.\left\{c_{1} \Rightarrow e_{1}|\ldots| c_{n} \Rightarrow e_{n}\right\}\right)_{\rho} \in$ $\llbracket \theta \rrbracket_{\pi, \xi}$. As $\left(\left[\text { case } e \text { of }\left\{c_{1} \Rightarrow e_{1}|\ldots| c_{n} \Rightarrow e_{n}\right\}\right)_{\rho}=\right.$ case $\left([e)_{\rho}\right.$ of $\left\{c_{1} \Rightarrow\left(\left[e_{1}\right]_{\rho}|\ldots|\right.\right.$ $c_{n} \Rightarrow\left(\left[e_{n}\right]_{\rho}\right\}$, this amounts to showing that case $\left([e)_{\rho}\right.$ of $\left\{c_{1} \Rightarrow\left(\left[e_{1}\right]_{\rho}|\ldots| c_{n} \Rightarrow\right.\right.$ $\left(\left[e_{n}\right)_{\rho}\right\} \in \llbracket \theta \rrbracket_{\pi, \xi}$.

As $(\pi, \xi, \rho) \models \Gamma$, from the induction hypothesis we get that $\left([e)_{\rho} \in \llbracket d^{\widehat{s}} \vec{\tau} \rrbracket_{\pi, \xi} \subseteq \mathrm{SN}\right.$ and $\left(\llbracket e_{k} \rrbracket_{\rho} \in \llbracket \operatorname{Inst}_{c_{k}}^{s} \quad \vec{\tau} \rightarrow \theta \rrbracket_{\pi, \xi}=\llbracket \operatorname{lnst}_{c_{k}}^{s} \vec{\tau} \rrbracket_{\pi, \xi} \rightarrow \llbracket \theta \rrbracket_{\pi, \xi} \subseteq \mathrm{SN}\right.$ for each $k \in 1 . . n$.

Since $\llbracket d^{\hat{s}} \vec{\tau} \rrbracket_{\pi, \xi}=\left\ulcorner c_{1} \llbracket \operatorname{lnst}_{c_{1}}^{s} \vec{\tau} \rrbracket_{\pi, \xi} \cup \ldots \cup c_{n} \llbracket \operatorname{lnst}_{c_{n}}^{s} \vec{\tau} \rrbracket_{\pi, \xi}\right\urcorner$, it must be the case that $\left(\left[e \rrbracket_{\rho} \rightarrow_{k} b\right.\right.$ for some $b \in$ Base $\cup c_{1} \llbracket \operatorname{lnst}_{c_{1}}^{s} \vec{\tau} \rrbracket_{\pi, \xi} \cup \ldots \cup c_{n} \llbracket \operatorname{lnst}_{c_{n}}^{s} \vec{\tau} \rrbracket_{\pi, \xi}$.

From $b \in$ Base $\cup c_{1} \llbracket \operatorname{lnst}_{c_{1}}^{s} \vec{\tau} \rrbracket_{\pi, \xi} \cup \ldots \cup c_{n} \llbracket \operatorname{lnst}_{c_{n}}^{s} \vec{\tau} \rrbracket_{\pi, \xi}$, it follows that case $b$ of $\left\{c_{1} \Rightarrow\right.$ $\left(\left[e_{1}\right)_{\rho}|\ldots| c_{n} \Rightarrow\left(\left[e_{n} \rrbracket_{\rho}\right\} \in \llbracket \theta \rrbracket_{\pi, \xi} \subseteq \mathrm{SN}\right.\right.$. Indeed, if $b \in$ Base, then case $b$ of $\left\{c_{1} \Rightarrow\right.$ $\left(\left[e_{1}\right]_{\rho}|\ldots| c_{n} \Rightarrow\left(\left[e_{n} \rrbracket_{\rho}\right\} \in\right.\right.$ Base $\subseteq \llbracket \theta \rrbracket_{\pi, \xi}$, as $\left(\left[e_{k}\right)_{\rho} \in \mathrm{SN}\right.$ for each $k \in 1 . . n$; if $b \in c_{k} \llbracket \operatorname{Inst}_{c_{k}}^{s} \quad \vec{\tau} \rrbracket_{\pi, \xi}$ for some $k \in 1 . . n$, then $b=c_{k} \vec{a}$ for some $\vec{a} \in \llbracket \operatorname{lnst}_{c_{k}}^{s} \vec{\tau} \rrbracket_{\pi, \xi}$ and therefore case $b$ of $\left\{c_{1} \Rightarrow\left(\mid e_{1}\right]_{\rho}|\ldots| c_{n} \Rightarrow\left(\left[e_{n}\right]_{\rho}\right\} \rightarrow_{k}\left(\left[e_{k}\right)_{\rho} \vec{a} \in \llbracket \theta \rrbracket_{\pi, \xi}\right.\right.$ and, by Lemma 3.15 , case $b$ of $\left\{c_{1} \Rightarrow\left(\left[e_{1}\right]_{\rho}|\ldots| c_{n} \Rightarrow\left(\left[e_{n}\right]_{\rho}\right\} \in \mathrm{SN}\right.\right.$, hence case $b$ of $\left\{c_{1} \Rightarrow\right.$ $\left(\left(e_{1} \rrbracket_{\rho}|\ldots| c_{n} \Rightarrow\left(\left[e_{n} \rrbracket_{\rho}\right\} \in \llbracket \theta \rrbracket_{\pi, \xi}\right.\right.\right.$ as $\llbracket \theta \rrbracket_{\pi, \xi}$ is saturated.

From $(e e)_{\rho} \rightarrow_{k} b$ it follows that case $(e e)_{\rho}$ of $\left\{c_{1} \Rightarrow\left(\left[e_{1} D_{\rho}|\ldots| c_{n} \Rightarrow\left(\left[e_{n}\right]_{\rho}\right\} \rightarrow_{k}\right.\right.\right.$ case $b$ of $\left\{c_{1} \Rightarrow\left(\left[e_{1}\right]_{\rho}|\ldots| c_{n} \Rightarrow\left(\left[e_{n}\right]_{\rho}\right\}\right.\right.$; further, by Lemma 3.14 , case $([e])_{\rho}$ of $\left\{c_{1} \Rightarrow\right.$ $\left(\left[e_{1} \rrbracket_{\rho}|\ldots| c_{n} \Rightarrow\left(\left[e_{n} \rrbracket_{\rho}\right\} \in \mathrm{SN}\right.\right.\right.$. Since $\llbracket \theta \rrbracket_{\pi, \xi}$ is saturated, we get that case $\left([e)_{\rho}\right.$ of $\left\{c_{1} \Rightarrow\right.$ $\left(\left(e_{1} \rrbracket_{\rho}|\ldots| c_{n} \Rightarrow\left(\left(e_{n} \rrbracket_{\rho}\right\} \in \llbracket \theta \rrbracket_{\pi, \xi}\right.\right.\right.$. 
(rec)Assume the last step is

$$
\frac{\Gamma, f: d^{\imath} \vec{\tau} \rightarrow \theta \vdash e: \widehat{d}^{\imath} \vec{\tau} \rightarrow \theta[\imath:=\widehat{\imath}] \quad \imath \operatorname{pos} \theta}{\Gamma \vdash(\text { letrec } f=e): d^{s} \vec{\tau} \rightarrow \theta[\imath:=s]} \text { and } \imath \text { fresh in } \Gamma, \vec{\tau}
$$

Suppose $(\pi, \xi, \rho) \models \Gamma$. We have to show that $([(\text { letrec } f=e)])_{\rho} \in \llbracket d^{s} \vec{\tau} \rightarrow \theta[\imath:=s] \rrbracket_{\pi, \xi}$. As $\llbracket d^{s} \vec{\tau} \rightarrow \theta[\imath:=s] \rrbracket_{\pi, \xi}=\llbracket d^{2} \vec{\tau} \rightarrow \theta \rrbracket_{\pi_{0}, \xi}=\llbracket d^{2} \vec{\tau} \rrbracket_{\pi_{0}, \xi} \rightarrow \llbracket \theta \rrbracket_{\pi_{0}, \xi}$ and $((\text { letrec } f=e))_{\rho}=$ (letrec $f=\left([e)_{\rho_{0}}\right)$ where $\pi_{0}=\pi\left(\imath:=\llbracket s \rrbracket_{\pi}\right)$ and $\rho_{0}=\rho(f:=f)$, this amounts to showing that (letrec $f=\left([e] \rho_{\rho_{0}}\right) a \in \llbracket \theta \rrbracket_{\pi_{0}, \xi}$ for any $a \in \llbracket d^{2} \vec{\tau} \rrbracket_{\pi_{0}, \xi}$.

As $\left(\pi_{0}, \xi, \rho_{0}\right) \models \Gamma$ and $\rho_{0}(f)=f \in \mathcal{V}_{\mathcal{E}} \subseteq \llbracket d^{\imath} \vec{\tau} \rightarrow \theta \rrbracket_{\pi_{0}, \xi}$, by the induction hypothesis we get that $([e])_{\rho_{0}} \in \llbracket d^{\imath} \vec{\tau} \rightarrow \theta[\imath:=\widehat{\imath}] \rrbracket_{\pi_{0}, \xi} \subseteq \mathrm{SN}$.

We prove our goal by induction on $\pi_{0}(\imath)$.

$\left(\pi_{0}(\imath)=0\right)$ Suppose $a \in \llbracket d^{\imath} \vec{\tau} \rrbracket_{\pi_{0}, \xi}=\ulcorner\emptyset\urcorner \subseteq$ SN. Then $a \rightarrow_{k} b$ for some $b \in$ Base.

Since $([e])_{\rho_{0}} \in \mathrm{SN}$, from $b \in$ Base it follows that (letrec $\left.f=([e])_{\rho_{0}}\right) b \in$ Base $\subseteq$ $\llbracket \theta \rrbracket_{\pi_{0}, \xi} \subseteq \mathrm{SN}$.

From $a \rightarrow_{k} b$ it follows that (letrec $\left.f=([e])_{\rho_{0}}\right) a \rightarrow_{k}$ (letrec $f=\left([e] \rho_{\rho_{0}}\right) b$ and, by Lemma 3.14, (letrec $\left.f=([e])_{\rho_{0}}\right) a \in \mathrm{SN}$.

Since $\llbracket \theta \rrbracket_{\pi_{0}, \xi}$ is a saturated set, we can conclude that (letrec $\left.f=(\llbracket e)_{\rho_{0}}\right) a \in \llbracket \theta \rrbracket_{\pi_{0}, \xi}$. $\left(\pi_{0}(\imath)=y+1\right)$ Let $\pi^{\prime}=\pi(\imath:=y)$ and $\rho^{\prime}=\rho\left(f:=\left(\right.\right.$ letrec $\left.f=\left([e] \rho_{0}\right)\right)$. As $\left(\pi^{\prime}, \xi, \rho^{\prime}\right) \models$ $\Gamma$ and as by the inner induction hypothesis $\rho^{\prime}(f)=\left(\right.$ letrec $\left.f=([e])_{\rho_{0}}\right) \in \llbracket d^{l} \vec{\tau} \rrbracket_{\pi^{\prime}, \xi} \rightarrow$ $\llbracket \theta \rrbracket_{\pi^{\prime}, \xi}=\llbracket d^{2} \vec{\tau} \rightarrow \theta \rrbracket_{\pi^{\prime}, \xi}$, by the outer induction hypothesis we get that $\left([e)_{\rho^{\prime}} \in\right.$ $\llbracket d^{\imath} \vec{\tau} \rightarrow \theta[\imath:=\widehat{\imath}] \rrbracket_{\pi^{\prime}, \xi}=\llbracket d^{\imath} \vec{\tau} \rightarrow \theta \rrbracket_{\pi_{0}, \xi}$.

Suppose $a \in \llbracket d^{2} \vec{\tau} \rrbracket_{\pi_{0}, \xi}=\left\ulcorner c_{1} \llbracket\right.$ Inst $_{c_{1}}^{\imath} \vec{\tau} \rrbracket_{\pi^{\prime}, \xi} \cup \ldots \cup c_{n} \llbracket$ Inst $\left._{c_{n}}^{\imath} \vec{\tau} \rrbracket_{\pi^{\prime}, \xi}\right\urcorner \subseteq$ SN. Then $a \rightarrow_{k} b$ for some $b \in$ Base $\cup c_{1} \llbracket \operatorname{Inst}_{c_{1}}^{i} \vec{\tau} \rrbracket_{\pi^{\prime}, \xi} \cup \ldots \cup c_{n} \llbracket \operatorname{Inst}_{c_{n}}^{l_{n}} \vec{\tau} \rrbracket_{\pi^{\prime}, \xi}$.

From $b \in$ Base $\cup c_{1} \llbracket$ Inst $_{c_{1}}^{\imath} \vec{\tau} \rrbracket_{\pi^{\prime}, \xi} \cup \ldots \cup c_{n} \llbracket$ Inst $_{c_{n}}^{\imath} \vec{\tau} \rrbracket_{\pi^{\prime}, \xi}$ we get that (letrec $f=$ $\left.([e])_{\rho_{0}}\right) b \in \llbracket \theta \rrbracket_{\pi_{0}, \xi} \subseteq \mathrm{SN}$. Indeed, if $b \in$ Base, then (letrec $f=\left([e)_{\rho_{0}}\right) b \in$ Base $\subseteq$ $\llbracket \theta \rrbracket_{\pi_{0}, \xi}$, since $\left([e)_{\rho_{0}} \in \mathrm{SN}\right.$; if $b \in c_{k} \llbracket \operatorname{lnst}_{c_{k}}^{2} \vec{\tau} \rrbracket_{\pi^{\prime}, \xi} \subseteq \llbracket d^{2} \vec{\tau} \rrbracket_{\pi_{0}, \xi}$ for some $k \in 1 . . n$, then (letrec $f=\left([e] \rho_{\rho_{0}}\right) b \rightarrow_{k}([e])_{\rho_{0}}\left[f:=\left(\right.\right.$ letrec $\left.\left.f=([e])_{\rho_{0}}\right)\right] b=([e])_{\rho^{\prime}} b \in \llbracket \theta \rrbracket_{\pi_{0}, \xi}$ and, by Lemma 3.15, (letrec $f=\left([e] \rho_{\rho_{0}}\right) b \in \mathrm{SN}$, hence (letrec $f=\left([e] \rho_{\rho_{0}}\right) b \in \llbracket \theta \rrbracket_{\pi_{0}, \xi}$ as $\llbracket \theta \rrbracket_{\pi_{0}, \xi}$ is saturated.

From $a \rightarrow_{k} b$ it follows that (letrec $\left.f=([e])_{\rho_{0}}\right) a \rightarrow_{k}$ (letrec $f=\left([e] \rho_{\rho_{0}}\right) b$ and, by Lemma 3.14, (letrec $\left.f=([e])_{\rho_{0}}\right) a \in \mathrm{SN}$.

Since $\llbracket \theta \rrbracket_{\pi_{0}, \xi}$ is a saturated set, we can conclude that (letrec $\left.f=(\llbracket e)_{\rho_{0}}\right) a \in \llbracket \theta \rrbracket_{\pi_{0}, \xi}$. $\left(\pi_{0}(\imath)=x\right.$ where $x$ is a limit ordinal)Suppose $a \in \llbracket d^{\imath} \vec{\tau} \rrbracket_{\pi_{0}, \xi}=\bigcup y<x . \llbracket d^{\imath} \vec{\tau} \rrbracket_{\pi(\imath:=y), \xi}$.

Then $a \in \llbracket d^{\imath} \vec{\tau} \rrbracket_{\pi(\imath:=y), \xi}$ for some $y<x$. By the inner induction hypothesis and by the positivity of $\imath$ in $\theta$, we therefore get that (letrec $\left.f=([e])_{\rho_{0}}\right) a \in \llbracket \theta \rrbracket_{\pi(\imath:=y), \xi} \subseteq$ $\llbracket \theta \rrbracket_{\pi_{0}, \xi} \cdot$

(sub) Assume the last step is

$$
\frac{\Gamma \vdash e: \sigma \quad \sigma \sqsubseteq \sigma^{\prime}}{\Gamma \vdash e: \sigma^{\prime}}
$$

Suppose $(\pi, \xi, \rho) \models \Gamma$. We have to show that $\left([e]_{\rho} \in \llbracket \sigma^{\prime} \rrbracket_{\pi, \xi}\right.$. As $(\pi, \xi, \rho) \models \Gamma$, the induction hypothesis gives $\left([e)_{\rho} \in \llbracket \sigma \rrbracket_{\pi, \xi}\right.$ and the subtyping soundness gives $\llbracket \sigma \rrbracket_{\pi, \xi} \subseteq$ $\llbracket \sigma^{\prime} \rrbracket_{\pi, \xi}$. Together, these give $\left([e]_{\rho} \in \llbracket \sigma^{\prime} \rrbracket_{\pi, \xi}\right.$. 
The main result of this subsection follows as an immediate corollary of the soundness of the typing system.

Proposition 3.36 (Strong normalization). $\rightarrow_{\beta \iota \mu}$ is strongly normalizing on typable expressions:

$$
\Gamma \vdash e: \sigma \text { derivable } \Rightarrow e \in \mathrm{SN}
$$

Proof. Assume $\Gamma \vdash e: \sigma$. Then, by Proposition 3.35, $\Gamma \models e: \sigma$. Consider a valuation $(\pi, \xi, \rho)$ where, for every $x \in \mathcal{V}_{\mathcal{E}}, \rho(x)=x$. For every $(x: \tau) \in \Gamma,\left([x)_{\rho}=x \in \llbracket \tau \rrbracket_{\pi, \xi}\right.$, since $\llbracket \tau \rrbracket_{\pi, \xi}$ is saturated, hence $(\pi, \xi, \rho) \models \Gamma$. Therefore $\left([e]_{\rho} \in \llbracket \sigma \rrbracket_{\pi, \xi}\right.$. As $\left([e)_{\rho}=e\right.$, we have

$$
e \in \llbracket \sigma \rrbracket_{\pi, \xi} \subseteq \mathrm{SN}
$$

\section{The System $\lambda_{\mathcal{G}}$}

In this section we present the system $\lambda_{\mathcal{G}}$, a simply typed $\lambda$-calculus with inductive types. The terms allowed in $\lambda_{\mathcal{G}}$ are the same as those allowed in $\lambda$. In particular, we continue to have the letrec constructor for defining functions recursively, but in $\lambda_{\mathcal{G}}$ (following what is done in (Giménez 1995)) termination of typable recursively defined functions is ensured by a syntactical condition $\mathcal{G}$ constraining uses of recursive calls in the body of definitions. The condition $\mathcal{G}$ is checked directly on the body of the function and not on its normal form because of the problem this would raise (as discussed in the introduction).

\subsection{The syntax of $\lambda_{\mathcal{G}}$}

The systems $\lambda_{\mathcal{G}}$ and $\lambda$ allow the same set of terms; they differ at the level of types in the following aspects:

1 stages are not present in $\lambda_{\mathcal{G}}$ and so datatypes are not annotated by stages;

2 in $\lambda_{\mathcal{G}}$ there is no subtyping relation;

3 the set of typing rules is different, and $\lambda_{\mathcal{G}}$ 's typing rule

$$
\frac{\Gamma, f: d \vec{\tau} \rightarrow \sigma \vdash e: d \vec{\tau} \rightarrow \sigma \quad \mathcal{G}_{f}^{x}(\emptyset, a)}{\Gamma \vdash(\text { letrec } f=e): d \vec{\tau} \rightarrow \sigma} \text { if } e \equiv \lambda x . a
$$

for letrec-expressions is complemented by the syntactical condition $\mathcal{G}$;

4 following (Giménez 1995), the datatypes allowed in $\lambda_{\mathcal{G}}$ are slightly more restricted than those of $\lambda^{\wedge}$ for, in the argument types of the constructors of a datatype, such datatype can only have strictly positive occurences; so throughout this section we assume that constructor schemes $(\delta, \vec{\alpha}, \vec{\sigma})$ are as in Definition 2.8 where condition 1 is replaced by the condition: each $\sigma_{i}$ is strictly positive w.r.t. $\delta$, or in other words, if $\delta$ occurs in $\sigma_{i}, \sigma_{i}$ is of the form $\vec{\gamma} \rightarrow \delta$ where $\vec{\gamma}$ has no occurrences of $\delta$.

Let us focus on the letrec operator and on the syntactical condition $\mathcal{G}$ it satisfies. This condition complements the reduction rule $\rightarrow_{\mu}$, ensuring that each expansion of the letrec operator consumes (at least) the constructor in the head of its argument. Informally, for a term (letrec $f=e$ ) we should have the following: 
$1 f$ may occur in $e$ only as the head of an application;

2 any application of $f$ must be protected by a case analysis of the formal argument of $e$, say $x$ (for this reason $f$ is said to be guarded by destructors); therefore $f$ must occur inside $e_{i}$ 's in the following context:

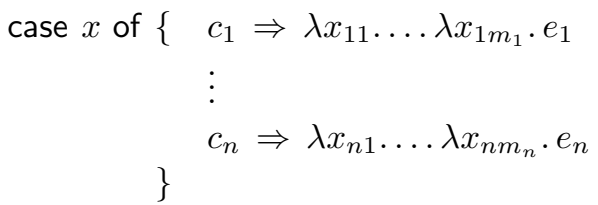

3 considering that the components of $x$ are the $x_{i j}$ (direct components) together with the components of each $x_{i j}$ (inner components), $f$ must be applied to a term of the form $z \vec{a}$ where $z$ is a recursive component of $x$ (i.e., $z$ is a component of $x$ whose type has occurrences of the type of $x$ ).

To illustrate the observations above, let us consider the examples already given in Section 2, plus and even, now transposed to $\lambda_{\mathcal{G}}$.

\section{Example 4.1.}

— The addition of two natural numbers.

$$
\begin{aligned}
& \text { (letrec } \quad \text { plus }=\lambda x . \lambda y \text {. case } x \text { of }\{0 \Rightarrow y \\
& \text { | s } \Rightarrow \lambda n . s(\text { plus ny) } \\
& ) \quad \text { Nat } \rightarrow \text { Nat } \rightarrow \text { Nat }
\end{aligned}
$$

Here the only application of plus is protected by a case analysis on $x$, the formal argument of plus. The argument of this application is the pattern variable $n$, a direct component of $x$.

- A function that indicates whether or not a natural number is even.

$$
\begin{aligned}
& \text { (letrec even }=\lambda x \text {. case } x \text { of }\{0 \Rightarrow \text { true } \\
& \mathrm{s} \Rightarrow \lambda y \text {. case } y \text { of }\{\mathrm{o} \Rightarrow \text { false } \\
& \mathrm{s} \Rightarrow \lambda z \text { even } z\} \\
& ): \quad \text { Nat } \rightarrow \text { Bool } \\
& \text { \} }
\end{aligned}
$$

In this example the application of even is guarded by a case analysis on the argument $x$. The argument of this application is the pattern variable $z$, an inner component of $x$ which becomes available in the case analysis on the pattern variable $y$, a direct component of $x$.

The formal description of the guarded-by-destructors condition is provided by the predicate $\mathcal{G}_{f}^{x}(V, a)$ defined below. The $V$ argument is a set of variables used to collect the pattern variables in $a$ representing the recursive components of $x$. In order to identify the recursive components of a variable, we start by characterizing the recursive positions of a constructor scheme as follows: 
1.

$$
\frac{f \neq y}{\mathcal{G}_{f}^{x}(U, y)} \quad \text { if } y \text { is a variable }
$$

2 .

$$
\frac{\mathcal{G}_{f}^{x}(U, a)}{\mathcal{G}_{f}^{x}(U, \lambda z . a)}
$$

3.

$$
\frac{\mathcal{G}_{f}^{x}(U, e)}{\mathcal{G}_{f}^{x}(U, \text { letrec } g=e)}
$$

4.

5. $\quad \frac{\mathcal{G}_{f}^{x}(U, a) \quad \mathcal{G}_{f}^{x}(U, b)}{\mathcal{G}_{f}^{x}(U, a b)}$

6.

$$
\frac{\mathcal{G}_{f}^{x}(U, z \vec{a})}{\mathcal{G}_{f}^{x}(U, f(z \vec{a}))} \quad \text { if } z \in U
$$

7. $\quad \frac{\mathcal{G}_{f}^{x}(U, e) \mathcal{G}_{f}^{x}\left(U, b_{i}\right) \quad(1 \leq i \leq n)}{\mathcal{G}_{f}^{x}\left(U, \text { case } e \text { of }\left\{c_{1} \Rightarrow b_{1}|\ldots| c_{n} \Rightarrow b_{n}\right\}\right)} \quad$ if $\left\{\begin{array}{c}e \not \equiv z \vec{a} \\ \vee \\ (e \equiv z \vec{a} \wedge z \notin U \cup\{x\})\end{array}\right.$

8. $\frac{\mathcal{G}_{f}^{x}\left(U, a_{j}\right) \quad(1 \leq j \leq m) \quad \mathcal{G}_{f}^{x}\left(V_{i}, e_{i}\right) \quad(1 \leq i \leq n)}{\mathcal{G}_{f}^{x}\left(U, \text { case }\left(z a_{1} \ldots a_{m}\right) \text { of }\left\{c_{1} \Rightarrow b_{1}|\ldots| c_{n} \Rightarrow b_{n}\right\}\right)}$

$$
\text { if }\left\{\begin{array}{l}
z \in U \cup\{x\} \\
b_{i} \equiv \lambda y_{1} \ldots \lambda y_{\operatorname{ar}\left(c_{i}\right) \cdot e_{i}} \\
V_{i} \equiv U \cup\left\{y_{j} \mid \operatorname{RP}\left(j, \mathrm{D}\left(c_{i}\right)\right) \text { for } 1 \leq j \leq \operatorname{ar}\left(c_{i}\right)\right\}
\end{array}\right.
$$

Fig. 6. Guarded-By-Destructors Rules

Definition 4.2. Let $c$ be a $\lambda_{\mathcal{G}}$ constructor such that $\mathrm{D}(c)=(\delta, \vec{\alpha}, \vec{\sigma})$. We say that the number $j$ corresponds to a recursive position of $\mathrm{D}(c)$, written $\operatorname{RP}(j, \mathrm{D}(c))$, if $\sigma_{j}$ is of the form $\vec{\gamma} \rightarrow \delta$.

The predicate $\mathcal{G}$ is now defined as follows:

Definition 4.3 ( $\mathcal{G}$ predicate). Let $U \subseteq \mathcal{V}$, let $x$ and $f$ be distinct variables not in $U$ and let $a \in \mathcal{E}$. The predicate $\mathcal{G}_{f}^{x}(U, a)$ is derivable using the rules in Figure 6 .

Lemma 4.4. If $f$ nocc $a$ then $\mathcal{G}_{f}^{x}(U, a)$.

Proof. By induction on the structure of $a$.

One can check that the guard predicate holds on addition. 
Example 4.5. The function plus of Example 4.1 can be shown guarded as follows

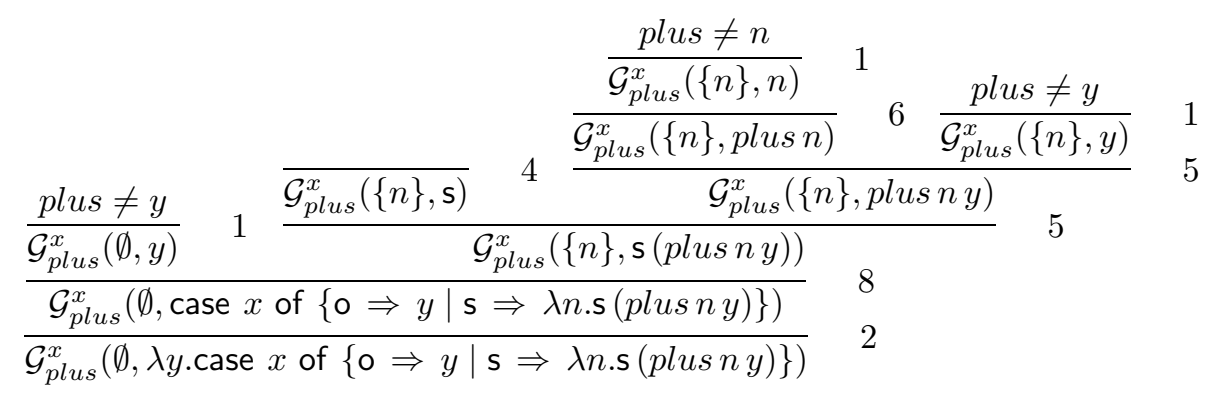

As suggested in the introduction, the predicate $\mathcal{G}$ is very sensitive to syntax. This is illustrated by the example below.

Example 4.6. Consider the following expression.

$$
\begin{aligned}
& \text { letrec plus }=\lambda x \text {. } \lambda y \text {. case } x \text { of }\{0 \Rightarrow y \\
& \text { | } \mathbf{s} \Rightarrow \lambda n . \mathbf{s}((\lambda g . g n y) p l u s)
\end{aligned}
$$

This expression also defines the addition of two natural numbers: it is obtained from the plus function defined in Example 4.1 by a $\beta$-expansion. However, this definition of plus does not satisfy condition $\mathcal{G}$ because the occurrence of plus in the letrec body is not the head of an application. So, when trying to prove the condition $\mathcal{G}$ we would have to derive $\mathcal{G}_{\text {plus }}^{x}(\{n\}$, plus $)$ which, by looking at the rules defining $\mathcal{G}$, we can immediately say to be underivable.

Another example of an expression not satisfying the guard condition is the Euclidean division already considered.

Example 4.7. The Euclidean division defined in Example 2.21 does not satisfy $\mathcal{G}$ for in the recursive call of the div function ( $\operatorname{div}$ (minus $\left.x^{\prime} y\right) y$ ) its argument (minus $x^{\prime} y$ ) is not a recursive component of its formal argument $(x)$, but instead the result of applying the previously defined minus to a recursive component $\left(x^{\prime}\right)$ of $x$.

We now turn to the typing system. First, one needs to define instances of constructors. The definition is almost identical to the one for $\lambda$, the only difference being the absence of stages.

Definition 4.8. Let $d \in \mathcal{D}, c \in \mathcal{C}(d)$ and $\vec{\tau} \in \mathcal{T}$ such that $\# \vec{\tau}=\operatorname{ar}(d)$. Assume $\mathrm{D}(c)=(\delta, \vec{\alpha}, \vec{\sigma})$. An instance of $c$ w.r.t. $\vec{\tau}$ is defined as follows

$$
\text { Inst }_{c} \vec{\tau}=\vec{\sigma}[\delta:=d \vec{\tau}][\vec{\alpha}:=\vec{\tau}]
$$

Typing of terms is defined in the usual way.

Definition 4.9 (Typing). The typing judgment $\Gamma \vdash e: \sigma$ is derivable if it can be inferred by the rules of Figure 7 , where $\mathcal{G}_{f}^{x}(\emptyset, a)$ is the guarded-by-destructors condition defined in Figure 6. 


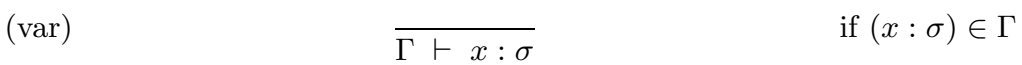

$$
\begin{aligned}
& \text { (abs) } \frac{\Gamma, x: \tau \vdash e: \sigma}{\Gamma \vdash \lambda x \cdot e: \tau \rightarrow \sigma} \\
& \text { (app) } \quad \frac{\Gamma \vdash e: \tau \rightarrow \sigma \quad \Gamma \vdash e^{\prime}: \tau}{\Gamma \vdash e e^{\prime}: \sigma} \\
& \text { (cons) } \overline{\Gamma \vdash c: \text { Inst }_{c} \vec{\tau} \rightarrow d \vec{\tau}} \quad \text { if } c \in \mathrm{C}(d) \\
& \text { (case) } \frac{\Gamma \vdash e: d \vec{\tau} \quad \Gamma \vdash e_{i}: \text { Inst }_{c_{i}} \vec{\tau} \rightarrow \theta \quad(1 \leq i \leq n)}{\Gamma \vdash \text { case } e \text { of }\left\{c_{1} \Rightarrow e_{1}|\ldots| c_{n} \Rightarrow e_{n}\right\}: \theta} \quad \text { if } \mathrm{C}(d)=\left\{c_{1}, \ldots, c_{n}\right\} \\
& \text { (rec) } \quad \frac{\Gamma, f: d \vec{\tau} \rightarrow \sigma \vdash e: d \vec{\tau} \rightarrow \sigma \quad \mathcal{G}_{f}^{x}(\emptyset, a)}{\Gamma \vdash(\text { letrec } f=e): d \vec{\tau} \rightarrow \sigma} \quad \text { if } e \equiv \lambda x . a
\end{aligned}
$$

Fig. 7. TYPING RULES FOR $\lambda_{\mathcal{G}}$

Below are presented some properties of $\lambda_{\mathcal{G}}$ used in the interpretation of $\lambda_{\mathcal{G}}$ into $\lambda^{\wedge}$ exhibited in the following section.

Lemma 4.10. $\Gamma_{1}, \Gamma_{2}, x: \tau, \Gamma_{3} \vdash a: \sigma \Rightarrow \Gamma_{1}, x: \tau, \Gamma_{2}, \Gamma_{3} \vdash a: \sigma$

Proof. By induction on the derivation of $\Gamma_{1}, \Gamma_{2}, x: \tau, \Gamma_{3} \vdash a: \sigma$.

Lemma 4.11 (Generation lemma for $\mathcal{G}$ ). If $\mathcal{G}_{f}^{x}(U, a)$ has a derivation $D$, then only one rule can be applied as the last step of $D$.

Proof. By case analysis on $a$. Note that only the conclusions of the rules 5 and 6 can be matched. Furthermore, in order to match the conclusions of such rules $a$ must be of the form $f(z \vec{b})$, in which case rule 5 cannot be applied as last rule for its left premise would be underivable.

Lemma 4.12. If $\mathcal{G}_{f}^{x}(U, a)$ and $U \subseteq V$, then $\mathcal{G}_{f}^{x}(V, a)$.

Proof. By induction on the derivation of $\mathcal{G}_{f}^{x}(U, a)$. The interesting case is when the last rule applied is rule 7 .

Assume $a \equiv$ case $e$ of $\left\{c_{1} \Rightarrow b_{1}|\ldots| c_{n} \Rightarrow b_{n}\right\}$ and the last step is

$$
\frac{\mathcal{G}_{f}^{x}(U, e) \quad \mathcal{G}_{f}^{x}\left(U, b_{i}\right) \quad(1 \leq i \leq n)}{\mathcal{G}_{f}^{x}\left(U, \text { case } e \text { of }\left\{c_{1} \Rightarrow b_{1}|\ldots| c_{n} \Rightarrow b_{n}\right\}\right)}
$$

- If $e \not \equiv z \vec{a}$ or if $e \equiv z a_{1} \ldots a_{m}, z \notin U \cup\{x\}$ and $z \notin V$, then by induction hypothesis $\mathcal{G}_{f}^{x}(V, e)$ and $\mathcal{G}_{f}^{x}\left(V, b_{i}\right)$ for $1 \leq i \leq n$, thus $\mathcal{G}_{f}^{x}(V, a)$ can be derived using rule 7 .

- Consider now $e \equiv z a_{1} \ldots a_{m}, z \notin U \cup\{x\}$ and $z \in V$. Each $b_{i}$ must be of the form $\lambda y_{1} \ldots \lambda y_{\operatorname{ar}\left(c_{i}\right)} . e_{i}$. Let $Q_{i} \equiv V \cup\left\{y_{j} \mid \operatorname{RP}\left(j, \mathrm{D}\left(c_{i}\right)\right)\right.$ for $\left.1 \leq j \leq \operatorname{ar}\left(c_{i}\right)\right\}$. For $1 \leq i \leq n$, since $V \subseteq Q_{i}$, using the induction hypothesis $\mathcal{G}_{f}^{x}\left(Q_{i}, b_{i}\right)$ and then, by Lemma 4.11, 
$\mathcal{G}_{f}^{x}\left(Q_{i}, e_{i}\right)$. Also, from the induction hypothesis we have $\mathcal{G}_{f}^{x}\left(V, a_{j}\right)$ for $1 \leq j \leq m$ and therefore, applying rule $8, \mathcal{G}_{f}^{x}(V, a)$.

The remaining cases can be easily proved using the induction hypothesis.

\section{Lemma 4.13 (Generation lemma for $\lambda_{\mathcal{G}}$ ).}

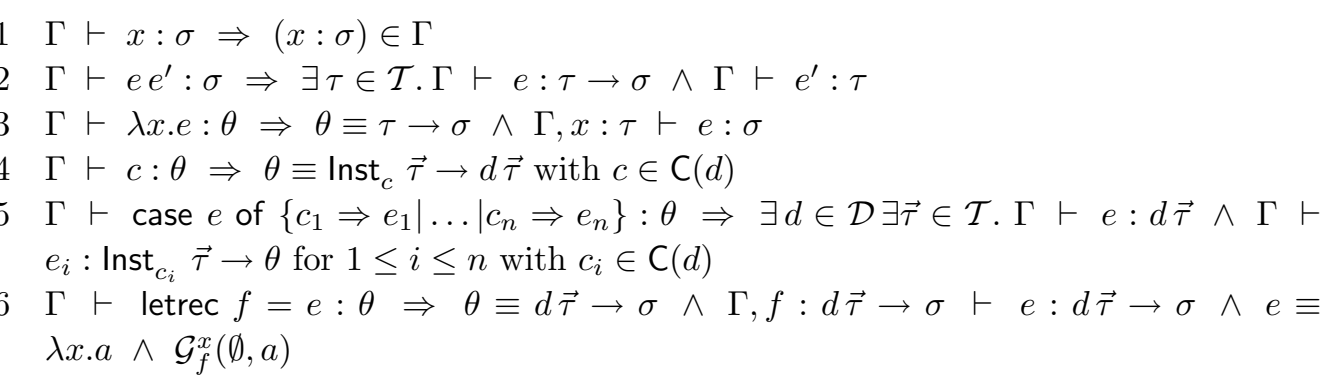

\subsection{From $\lambda_{\mathcal{G}}$ to $\lambda^{\Upsilon}$}

In this section we show that $\lambda \uparrow$ is a more general system than $\lambda_{\mathcal{G}}$. The Examples 4.6 and 4.7 already illustrated that some terms typable in $\lambda^{\wedge}$ cannot be typed in $\lambda_{\mathcal{G}}$. In this section we show that: if $\Gamma \vdash_{\lambda_{\mathcal{G}}} a: \sigma$ then $\Gamma \vdash_{\chi} a: \sigma$ (the subscript at the turnstyle sign indicating the type system considered). Naturally, the main difficulty in transiting from $\lambda_{\mathcal{G}}$ to $\lambda$ is posed by letrec-expressions because the two systems have different kinds of typing rules for these expressions.

Given $\Gamma \vdash_{\lambda_{\mathcal{G}}}$ letrec $f=\lambda x . a: d \vec{\tau} \rightarrow \sigma$, by the generation lemma for $\lambda_{\mathcal{G}}$, we have

$$
\Gamma, f: d \vec{\tau} \rightarrow \sigma, x: d \vec{\tau} \vdash_{\lambda_{\mathcal{G}}} a: \sigma \wedge \mathcal{G}_{f}^{x}(\emptyset, a)
$$

However, we would want to have

$$
\Gamma, f: d^{\imath} \vec{\tau} \rightarrow \sigma, x: \hat{d}^{\hat{\imath}} \vec{\tau} \vdash_{x} a: \sigma \quad(\imath \text { fresh in } \Gamma, \vec{\tau})
$$

in order to use the $\lambda^{\wedge}$ rec-rule and so derive $\Gamma \vdash_{x}$ letrec $f=\lambda x . a: d \vec{\tau} \rightarrow \sigma$. Intuitively $(6)$ is sufficient to guarantee (7) because, as we have $\mathcal{G}_{f}^{x}(\emptyset, a)$, all the possible occurrences of $f$ in $a$ are of the form $f(z \vec{a})$, with $z$ being a recursive component of $x$. In (7) we have $x: d^{\widehat{\imath}} \vec{\tau}$ so, if $z$ is a recursive component of $x$ we should have $z: \vec{\gamma} \rightarrow d^{2} \vec{\tau}$. Hence $f(z \vec{a})$ is also typable in $\lambda^{\Upsilon}$.

The remainder of this subsection is devoted to the embedding of $\lambda_{\mathcal{G}}$ into $\lambda^{\Upsilon}$. In this embedding the Main Lemma below plays a central role. There we present the full construction underlying the lemma because it lays open the details of the relation between the systems $\lambda_{\mathcal{G}}$ and $\lambda^{\wedge}$.

In the following we assume that each variable $x_{i}$ is uniquely associated to a stage variable $\jmath_{i}$. Recall also that, in $\lambda$, the notation $d \vec{\tau}$ abbreviates the datatype $d^{\infty} \vec{\tau}$. 
Lemma 4.14 (Main Lemma). Let

$$
\begin{array}{rlrl}
\Gamma_{0} & =\Gamma & \\
\Gamma_{i}=\Gamma_{i-1}, f_{i}: d_{i} \overrightarrow{\tau_{i}} \rightarrow \sigma_{i}, x_{i}: d_{i} \overrightarrow{\tau_{i}} & & \text { for } 1 \leq i \leq n \\
\widehat{\Gamma_{0}}=\Gamma_{0} & \\
\widehat{\Gamma_{i}}=\widehat{\Gamma_{i-1}}, f_{i}: d_{i}^{j_{i}} \overrightarrow{\tau_{i}} \rightarrow \sigma_{i}, x_{i}: d_{i}^{\widehat{\jmath_{i}}} \overrightarrow{\tau_{i}} & & \text { for } 1 \leq i \leq n \\
& & \text { where } \jmath_{i} \text { is a fresh stage variable } & \text { associated to } x_{i}
\end{array}
$$

and, for $1 \leq i \leq n$, let $U_{i}$ be a set of variables such that for each $z \in U_{i}, z: \vec{\gamma} \rightarrow d_{i} \vec{\tau}_{i} \in \Gamma$ and so that all the $U_{i}$ 's are disjoint. Then,

$$
\Gamma_{n} \vdash_{\lambda_{\mathcal{G}}} a: \sigma \wedge\left(\forall i \in\{1, \ldots, n\} \cdot \mathcal{G}_{f_{i}}^{x_{i}}\left(U_{i}, a\right)\right) \Rightarrow\left[\widehat{\Gamma_{n}}\right]_{U} \vdash_{X} a: \sigma
$$

where $U=\bigcup_{1 \leq i \leq n} U_{i}$ and $\left[\widehat{\Gamma_{n}}\right]_{U}$ is obtained from $\widehat{\Gamma_{n}}$ by replacing each declaration $z: \vec{\gamma} \rightarrow d_{i} \vec{\tau}_{i}$ (with $z \in U_{i}$ ) by $z: \vec{\gamma} \rightarrow d_{i}^{j_{i}} \vec{\tau}_{i}$. Note that in order to make $\Gamma_{n}$ a context, in particular, all the $f_{i}$ 's and $x_{i}$ 's must be distinct and cannot be declared in $\Gamma$.

Proof. By induction on the structure of $a$.

1 Case $a \equiv x$, the hypothesis is

$$
\Gamma_{n} \vdash_{\lambda_{\mathcal{G}}} x: \sigma \wedge \forall i \in\{1, \ldots, n\} \cdot \mathcal{G}_{f_{i}}^{x_{i}}\left(U_{i}, x\right)
$$

— If $x \equiv x_{i}$ for some $i \in\{1, \ldots, n\}, \sigma \equiv d_{i} \overrightarrow{\tau_{i}}$ and so, $\left[\widehat{\Gamma_{n}}\right]_{U} \quad \vdash_{x} \quad x: d_{i}^{\widehat{j_{i}}} \overrightarrow{\tau_{i}}$. As $d_{i}^{\widehat{\jmath_{i}}} \vec{\tau}_{i} \sqsubseteq d_{i}^{\infty} \vec{\tau}_{i}$, using the rule (sub),

$$
\left[\widehat{\Gamma_{n}}\right]_{U} \vdash_{x} x: d_{i} \overrightarrow{\tau_{i}}
$$

— If $x \not \equiv x_{i}$ for every $i \in\{1, \ldots, n\}$ then, since $\forall i \in\{1, \ldots, n\} . \mathcal{G}_{f_{i}}^{x_{i}}\left(U_{i}, x\right), x \not \equiv f_{i}$ for every $i \in\{1, \ldots, n\}$. Therefore, using Lemma 4.13, $(x: \sigma) \in \Gamma$. Hence

(a) If $x \notin U$, then $\left[\widehat{\Gamma_{n}}\right]_{U} \vdash_{x} x: \sigma$.

(b) If $x \in U$ then, $\sigma \equiv \vec{\gamma} \rightarrow d_{i} \vec{\tau}_{i}$ for some $i \in\{1, \ldots, n\}$. So, $\left[\widehat{\Gamma_{n}}\right]_{U} \vdash_{x} x: \vec{\gamma} \rightarrow$ $d_{i}^{J_{i}} \vec{\tau}_{i}$ and, since $\vec{\gamma} \rightarrow d_{i}^{j_{i}} \vec{\tau}_{i} \sqsubseteq \vec{\gamma} \rightarrow d_{i}^{\infty} \overrightarrow{\tau_{i}}$, by (sub)

$$
\left[\widehat{\Gamma_{n}}\right]_{U} \vdash_{x} x: \sigma
$$

2 Case $a \equiv e e^{\prime}$ the hypothesis is

$$
\Gamma_{n} \vdash_{\lambda_{\mathcal{G}}} e e^{\prime}: \sigma \wedge \forall i \in\{1, \ldots, n\} \cdot \mathcal{G}_{f_{i}}^{x_{i}}\left(U_{i}, e e^{\prime}\right)
$$

- If $e \equiv f_{i}$ for some $i \in\{1, \ldots, n\}$ then, by Lemma $4.11, e^{\prime} \equiv z \vec{b}, \mathcal{G}_{f_{i}}^{x_{i}}\left(U_{i}, e^{\prime}\right)$ and $z \in U_{i}$. Moreover:

(a) $\Gamma_{n} \vdash_{\lambda_{\mathcal{G}}} f_{i}: d_{i} \overrightarrow{\tau_{i}} \rightarrow \sigma_{i}$ and $\sigma \equiv \sigma_{i}$. So, $\left[\widehat{\Gamma_{n}}\right]_{U} \vdash_{\chi} f_{i}: d_{i}^{\jmath_{i}} \overrightarrow{\tau_{i}} \rightarrow \sigma$.

(b) $\Gamma_{n} \vdash_{\lambda_{\mathcal{G}}} z: \vec{\gamma} \rightarrow d_{i} \overrightarrow{\tau_{i}}$. So, $\left[\widehat{\Gamma_{n}}\right]_{U} \vdash_{x} z: \vec{\gamma} \rightarrow d_{i}^{J_{i}} \vec{\tau}_{i}$ because $z \in U_{i}$.

(c) $\Gamma_{n} \vdash_{\lambda_{\mathcal{G}}} \vec{b}: \vec{\gamma}$ (using this notation to abbreviate the list of judgments $\Gamma_{n} \vdash_{\lambda_{\mathcal{G}}}$ $b_{k}: \gamma_{k}$ for each $\left.b_{k} \in \vec{b}\right)$ and for every $b_{k} \in \vec{b}, \mathcal{G}_{f_{i}}^{x_{i}}\left(U_{i}, b_{k}\right)$ because $z \neq f_{i}$. For $j \in\{1, \ldots, n\}-\{i\}, e \neq f_{j}$ and, by Lemma $4.11, \mathcal{G}_{f_{j}}^{x_{j}}\left(U_{j}, b_{k}\right)$ for every $b_{k} \in \vec{b}$. 
Therefore, by induction hypothesis,

$$
\left[\widehat{\Gamma_{n}}\right]_{U} \vdash_{x} \vec{b}: \vec{\gamma}
$$

From (a), (b) and (c), using (app), one can then obtain

$$
\left[\widehat{\Gamma_{n}}\right]_{U} \vdash_{x} f_{i}(z \vec{b}): \sigma
$$

— If $e \not \equiv f_{i}$ for every $i \in\{1, \ldots, n\}$ then, using Lemmas 4.11 and 4.13,

$$
\Gamma_{n} \vdash_{\lambda_{\mathcal{G}}} e: \gamma \rightarrow \sigma \wedge \Gamma_{n} \vdash_{\lambda_{\mathcal{G}}} e^{\prime}: \gamma
$$

and

$$
\forall i \in\{1, \ldots, n\} . \mathcal{G}_{f_{i}}^{x_{i}}\left(U_{i}, e\right) \wedge \mathcal{G}_{f_{i}}^{x_{i}}\left(U_{i}, e^{\prime}\right)
$$

Hence, by induction hypothesis,

$$
\left[\widehat{\Gamma_{n}}\right]_{U} \vdash_{x} e: \gamma \rightarrow \sigma \wedge\left[\widehat{\Gamma_{n}}\right]_{U} \vdash_{x} e^{\prime}: \gamma
$$

Using the rule (app) one obtains $\left[\widehat{\Gamma_{n}}\right]_{U} \vdash_{\chi} e e^{\prime}: \sigma$.

3 Case $a \equiv \lambda y$.e, the hypothesis is

$$
\Gamma_{n} \vdash_{\lambda_{\mathcal{G}}} \lambda y . e: \sigma \wedge \forall i \in\{1, \ldots, n\} . \mathcal{G}_{f_{i}}^{x_{i}}\left(U_{i}, \lambda y . e\right)
$$

Using Lemmas 4.13 and $4.10, \sigma \equiv \gamma \rightarrow \sigma^{\prime}$ for some $\gamma, \sigma^{\prime} \in \mathcal{T}$ and also

$$
\Gamma, y: \gamma, f_{1}: d_{1} \overrightarrow{\tau_{1}} \rightarrow \sigma_{2}, x_{1}: d_{1} \overrightarrow{\tau_{1}}, \ldots, f_{n}: d_{n} \overrightarrow{\tau_{n}} \rightarrow \sigma, x_{n}: d_{n} \overrightarrow{\tau_{n}} \vdash_{\lambda_{\mathcal{G}}} e: \sigma^{\prime}
$$

By Lemma 4.11, $\forall i \in\{1, \ldots, n\} . \mathcal{G}_{f_{i}}^{x_{i}}\left(U_{i}, e\right)$. Hence, by induction hypothesis,

$$
\left[\Gamma, y: \gamma, f_{1}: d_{1}^{\jmath_{1}} \overrightarrow{\tau_{1}} \rightarrow \sigma_{1}, x_{1}: d_{1}^{\widehat{\jmath_{1}}} \overrightarrow{\tau_{1}}, \ldots, f_{n}: d_{n}^{\jmath_{n}} \overrightarrow{\tau_{n}} \rightarrow \sigma_{n}, x_{n}: \widehat{d_{n}^{\jmath_{n}}} \overrightarrow{\tau_{n}}\right]_{U} \vdash x e: \sigma^{\prime}
$$

We know that $y \notin \Gamma$ and so, $y \notin U$. Therefore, using Lemma $3.6\left[\widehat{\Gamma_{n}}\right]_{U}, y: \gamma \vdash_{x} e: \sigma^{\prime}$ and the proof of this case is concluded applying rule (abs).

4 Case $a \equiv c$ and $c \in \mathrm{C}(d)$, one assumes $\Gamma_{n} \vdash_{\lambda_{\mathcal{G}}} c:$ Inst $_{c} \vec{\tau} \rightarrow d \vec{\tau}$. Thus in $\hat{\lambda}$ one can apply (cons) to obtain $\left[\widehat{\Gamma_{n}}\right]_{U} \vdash_{x} c:$ Inst $_{c}^{\infty} \vec{\tau} \rightarrow d^{\infty} \vec{\tau}$, and since, Inst ${ }_{c} \vec{\tau}$ is being used as an abbreviation for $\operatorname{Inst}_{c}^{\infty} \vec{\tau}$ and $d^{\widehat{\infty}} \vec{\tau} \sqsubseteq d^{\infty} \vec{\tau}$, one also has

$$
\left.\widehat{\Gamma_{n}}\right]_{U} \vdash_{\chi} c: \operatorname{lnst}_{c} \vec{\tau} \rightarrow d \vec{\tau}
$$

5 Case $a \equiv$ case $e$ of $\left\{c_{1} \Rightarrow b_{1}|\ldots| c_{m} \Rightarrow b_{m}\right\}$, the hypotheses are

$$
\begin{array}{r}
\Gamma_{n} \vdash_{\lambda_{\mathcal{G}}} \text { case } e \text { of }\{\vec{c} \Rightarrow \vec{b}\}: \sigma \\
\forall i \in\{1, \ldots, n\} . \mathcal{G}_{f_{i}}^{x_{i}}\left(U_{i} \text {, case } e \text { of }\{\vec{c} \Rightarrow \vec{b}\}\right)
\end{array}
$$

and from (8), applying Lemma 4.13, there exists $d, \vec{\tau}$ such that

$$
\begin{aligned}
& \Gamma_{n} \vdash_{\lambda_{\mathcal{G}}} e: d \vec{\tau} \\
& \Gamma_{n} \vdash_{\lambda_{\mathcal{G}}} b_{k}: \text { Inst }_{c_{k}} \vec{\tau} \rightarrow \sigma
\end{aligned}
$$

for each $1 \leq k \leq m$. Two cases can now occur.

- If $e \not \equiv z \vec{a}$ or $e \equiv z \vec{a}$ and $z \notin U_{i} \cup\left\{x_{i}\right\}$ for every $i \in\{1, \ldots, n\}$, then from (9) by Lemma 4.11

$$
\forall i \in\{1, \ldots, n\} . \forall k \in\{1, \ldots, m\} . \mathcal{G}_{f_{i}}^{x_{i}}\left(U_{i}, e\right) \wedge \mathcal{G}_{f_{i}}^{x_{i}}\left(U_{i}, b_{k}\right)
$$


Thus applying the induction hypothesis to (10), followed by rule (sub), one has $\left[\widehat{\Gamma_{n}}\right]_{U} \vdash_{\chi} e: d^{\widehat{\infty}} \vec{\tau}$ and applying the induction hypothesis to (11) one obtains $\left[\widehat{\Gamma_{n}}\right]_{U} \vdash_{x} \quad b_{k}:$ Inst $_{c_{k}}^{\infty} \vec{\tau} \rightarrow \sigma$. Derivations of these judgments can now be put together by means of the rule (case), proving

$$
\left[\widehat{\Gamma_{n}}\right]_{U} \vdash_{X} \text { case } e \text { of }\{\vec{c} \Rightarrow \vec{b}\}: \sigma
$$

- Consider now that $e \equiv z \vec{a}$ and $z \in U_{i} \cup\left\{x_{i}\right\}$ for some $i \in\{1, \ldots, n\}$ (recall that such $i$ must be unique since: the $U_{j}$ 's are disjoint and contain none of the $x_{j}$ 's; and the $x_{j}$ 's are distinct). Let, for each $1 \leq k \leq m$,

$$
\left\{\begin{array}{l}
b_{k} \equiv \lambda \overrightarrow{y_{k}} \cdot e_{k} \\
V_{k, i} \equiv U_{i} \cup\left\{y_{k, r} \mid \operatorname{RP}\left(r, \mathrm{D}\left(c_{k}\right)\right) \text { for } 1 \leq r \leq \operatorname{ar}\left(c_{k}\right)\right\} \\
V_{k, j} \equiv U_{j} \text { for } j \in\{1, \ldots, n\}-\{i\} \\
V_{k} \equiv \bigcup_{1 \leq j \leq n} V_{k, j}
\end{array}\right.
$$

where $y_{k, r}$ denotes the $r$-th component of vector $\overrightarrow{y_{k}}$. Applying Lemma 4.11 to (9), one can now assume that for each $1 \leq j \leq n$

$$
\forall a_{s} \in \vec{a} \cdot \mathcal{G}_{f_{j}}^{x_{j}}\left(U_{j}, a_{s}\right) \wedge \forall k \in\{1, \ldots, m\} \cdot \mathcal{G}_{f_{j}}^{x_{j}}\left(V_{k, j}, e_{k}\right)
$$

From (11) by Lemmas 4.13 and 4.10 , one has

$$
\Gamma, \overrightarrow{y_{k}}: \text { Inst }_{c_{k}} \vec{\tau}, \Gamma_{n} \backslash \Gamma \vdash_{\lambda_{\mathcal{G}}} e_{k}: \sigma
$$

where $\Gamma_{n} \backslash \Gamma$ is the context $\Gamma_{n}$ without the declarations in $\Gamma$. Moreover, $y_{k, r}$ : $\gamma_{y_{k, r}} \rightarrow d_{i} \overrightarrow{\tau_{i}} \in\left(\overrightarrow{y_{k}}:\right.$ Inst $\left._{c_{k}} \vec{\tau}\right)$ for each $1 \leq r \leq \operatorname{ar}\left(c_{k}\right)$ such that $\operatorname{RP}\left(r, \mathrm{D}\left(c_{k}\right)\right)$ and thus, for each $1 \leq k \leq m$ and $1 \leq j \leq n$, and for each $z \in V_{k, j}$ we have $z: \overrightarrow{\gamma_{z}} \rightarrow d_{i} \vec{\tau}_{i} \in\left(\Gamma, \overrightarrow{y_{k}}:\right.$ Inst $\left._{c_{k}} \vec{\tau}\right)$. Hence, by the induction hypothesis

$$
\left[\Gamma, \overrightarrow{y_{k}}: \widehat{\operatorname{lnst}_{c_{k}}} \vec{\tau}, \Gamma_{n} \backslash \Gamma\right]_{V_{k}} \vdash_{\chi} e_{k}: \sigma
$$

from which one can show $\left[\widehat{\Gamma_{n}}\right]_{U}, \overrightarrow{y_{k}}:$ Inst $_{c_{k}}^{\jmath_{i}} \vec{\tau} \vdash_{\chi} e_{k}: \sigma$ (observe that $V_{k}=$ $U \cup\left\{y_{k, r} \mid \operatorname{RP}\left(r, \mathrm{D}\left(c_{k}\right)\right)\right.$ for $\left.\left.1 \leq r \leq \operatorname{ar}\left(c_{k}\right)\right\}\right)$ and therefore, by the rule (abs), $\left.\widehat{\Gamma_{n}}\right]_{U} \vdash_{\chi} b_{k}:$ Inst $_{c_{k}}^{\jmath_{i}} \vec{\tau} \rightarrow \sigma$ holds.

To conclude the proof of this case, it suffices now to show that

$$
\left.\widehat{\left[\Gamma_{n}\right.}\right]_{U} \vdash_{\chi} e: d_{i}^{\widehat{\jmath_{i}}} \overrightarrow{\tau_{i}}
$$

and to use then the rule (case). In order to prove (13) one proceeds as follows.

(a) Case $e \equiv x_{i},\left[\widehat{\Gamma_{n}}\right]_{U} \vdash_{\chi} x_{i}: d_{i}^{\widehat{\jmath_{i}}} \overrightarrow{\tau_{i}}$ is derivable.

(b) Case $e \equiv z \vec{a}$ with $z \in U_{i}$, from (10) by Lemma 4.13, $\Gamma_{n} \vdash_{\lambda_{\mathcal{G}}} z: \vec{\gamma} \rightarrow d \vec{\tau}$ (thus, $d \vec{\tau} \equiv d_{i} \overrightarrow{\tau_{i}}$ ) and

$$
\Gamma_{n} \vdash_{\lambda_{\mathcal{G}}} \vec{a}: \vec{\gamma}
$$

Now, since (12) holds, one can apply the induction hypothesis to (14) obtaining $\left[\widehat{\Gamma_{n}}\right]_{U} \vdash_{\chi} \vec{a}: \vec{\gamma}$. It is also true that $z: \vec{\gamma} \rightarrow d_{i}^{\jmath_{i}} \overrightarrow{\tau_{i}} \in\left[\widehat{\Gamma_{n}}\right]_{U}$, for $z \in U_{i}$, and since $\vec{\gamma} \rightarrow d_{i}^{\jmath_{i}} \overrightarrow{\tau_{i}} \sqsubseteq \vec{\gamma} \rightarrow d_{i}^{\widehat{\jmath}_{i}} \overrightarrow{\tau_{i}}$, by (sub) and (app), $\left[\widehat{\Gamma_{n}}\right]_{U} \vdash_{x} z \vec{a}: d_{i}^{\widehat{\jmath_{i}}} \overrightarrow{\tau_{i}}$ holds. 
6 Case $a \equiv$ letrec $f=\lambda x \cdot a^{\prime}$, we must have $\sigma \equiv d \vec{\tau} \rightarrow \sigma^{\prime}$ for some $d \vec{\tau}, \sigma^{\prime} \in \mathcal{T}$, and the hypothesis is

$$
\Gamma_{n} \vdash_{\lambda_{\mathcal{G}}} \text { letrec } f=\lambda x \cdot a^{\prime}: d \vec{\tau} \rightarrow \sigma^{\prime} \wedge \forall i \in\{1, \ldots, n\} . \mathcal{G}_{f_{i}}^{x_{i}}\left(U_{i} \text {, letrec } f=\lambda x . a^{\prime}\right)
$$

By Lemma 4.13 we get

$$
\Gamma_{n}, f: d \vec{\tau} \rightarrow \sigma^{\prime}, x: d \vec{\tau} \vdash_{\lambda_{\mathcal{G}}} a^{\prime}: \sigma^{\prime}
$$

and $\mathcal{G}_{f}^{x}\left(\emptyset, a^{\prime}\right)$. Again by the hypothesis, by Lemma 4.11, $\forall i \in\{1, \ldots, n\} . \mathcal{G}_{f_{i}}^{x_{i}}\left(U_{i}, a^{\prime}\right)$ hence, assuming $U_{n+1}=\emptyset, x_{n+1}=x$ and $f_{n+1}=f$, we have

$$
\forall i \in\{1, \ldots, n+1\} . \mathcal{G}_{f_{i}}^{x_{i}}\left(U_{i}, a^{\prime}\right)
$$

So, by induction hypothesis $\left[\widehat{\Gamma_{n+1}}\right]_{U} \vdash_{x} a^{\prime}: \sigma^{\prime}$. Applying (abs) and (rec) we get $\left[\widehat{\Gamma_{n}}\right]_{U} \vdash_{x}$ letrec $f=\lambda x \cdot a^{\prime}:\left(d^{2} \vec{\tau} \rightarrow \sigma^{\prime}\right)[\imath:=\infty]$. Hence, for $\imath$ has no occurrences in $\vec{\tau}$ nor in $\sigma^{\prime}$,

$$
\left[\widehat{\Gamma_{n}}\right]_{U} \vdash_{x}\left(\text { letrec } f=\lambda x \cdot a^{\prime}\right): d \vec{\tau} \rightarrow \sigma^{\prime}
$$

We are now ready to prove the main result of this section.

\section{Proposition 4.15.}

$$
\Gamma \vdash_{\lambda_{\mathcal{G}}} a: \sigma \Rightarrow \Gamma \vdash_{x} a: \sigma
$$

Proof. By induction on the derivation of $\Gamma \vdash_{\lambda_{\mathcal{G}}} a: \sigma$.

(rec)Assume the last step is

$$
\frac{\Gamma, f: d \vec{\tau} \rightarrow \sigma \vdash_{\lambda_{\mathcal{G}}} e: d \vec{\tau} \rightarrow \sigma \quad \mathcal{G}_{f}^{x}(\emptyset, a)}{\Gamma \vdash_{\lambda_{\mathcal{G}}}(\text { letrec } f=e): d \vec{\tau} \rightarrow \sigma} \text { with } e \equiv \lambda x . a
$$

By Lemma 4.13, $\Gamma, f: d \vec{\tau} \rightarrow \sigma, x: d \vec{\tau} \vdash_{\lambda_{\mathcal{G}}} a: \sigma$ and since $\mathcal{G}_{f}^{x}(\emptyset, a)$ we are in conditions of applying the Main Lemma and conclude $\Gamma, f: d^{2} \vec{\tau} \rightarrow \sigma, x: d^{\hat{\imath}} \vec{\tau} \vdash_{x} a: \sigma$. Hence, applying the rules (abs) and (rec) one derives $\Gamma \vdash_{x}$ (letrec $\left.f=e\right):\left(d^{2} \vec{\tau} \rightarrow\right.$ $\sigma)[\imath:=\infty]$ which is the same as

$$
\Gamma \vdash_{x}(\text { letrec } f=e): d \vec{\tau} \rightarrow \sigma
$$

for $\imath$ does not occur in $\sigma$ or $\vec{\tau}$.

All the remaining cases can be easily proved using the induction hypothesis.

\section{Extension to coinductive types}

Coinductive types are a mechanism for the introduction of infinite objects into type theory, and are useful in the modelling of perpetual computations, e.g., the operation of process systems. The system $\lambda$ is readily extensible to support also coinductive types. We shall here outline the syntax of an appropriate extension of $\lambda$ and give some programming examples. 
First, the definition of the set $\mathcal{E}$ of terms is extended with corecursive definitions.

$$
e::=\ldots \mid\left({ }^{\text {co }} \text { letrec } f=e\right)
$$

In addition to $\beta$-, $\iota$-, and $\mu$-reduction, we define $\nu$-reduction as the compatible closure of the rule

$$
\begin{aligned}
& \text { case }\left({ }^{\text {co }} \text { letrec } f=e\right) \vec{a} \text { of }\left\{c_{1} \Rightarrow e_{1}|\ldots| c_{n} \Rightarrow e_{n}\right\} \\
& \quad \rightarrow \nu \quad \text { case } e\left[f:=\left({ }^{\text {co }} \text { letrec } f=e\right)\right] \vec{a} \text { of }\left\{c_{1} \Rightarrow e_{1}|\ldots| c_{n} \Rightarrow e_{n}\right\}
\end{aligned}
$$

The form of the $\nu$-reduction rule may look unexpected, but is dual to $\mu$-reduction: while the $\mu$-reduction rule allows unfolding of a recursive definition provided that the argument value is produced by a constructor, the $\nu$-reduction rule allows it, if the result value is consumed by a case-expression.

Second, the definition of the set $\mathcal{T}$ of type expressions is extended with codatatype approximation expressions

$$
\sigma, \tau::=\ldots \mid{ }^{\text {co }} d^{s} \vec{\tau}
$$

${ }^{\mathrm{co}} d^{\infty} \vec{\tau}$ will also be written as ${ }^{\mathrm{co}} d \vec{\tau}$.

The subtyping rules are supplemented with the following (codata) rule, dual to the (data) rule:

$$
\text { (codata) } \frac{s \preccurlyeq r \quad \tau_{i} \sqsubseteq \tau_{i}^{\prime} \quad(1 \leq i \leq \operatorname{ar}(d))}{{ }^{\mathrm{co}} d^{r} \vec{\tau} \sqsubseteq{ }^{{ }^{c o}} d^{s} \vec{\tau}}
$$

The typing rules are supplemented with the rules (cons'), (case') and (corec). The two first of these are essentially the same as the rules (cons), (case), but they are used for the construction and destruction of values of coinductive, not inductive types. Below

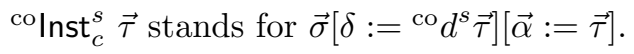

$$
\begin{aligned}
& \text { (cons') } \\
& \overline{\Gamma \vdash c:{ }^{\mathrm{co}} \operatorname{Inst}_{c}^{s} \vec{\tau} \rightarrow{ }^{\mathrm{co}} d^{\widehat{s}} \vec{\tau}} \\
& \text { (case') } \frac{\Gamma \vdash e^{\prime}:{ }^{\text {co }} d^{\widehat{s}} \vec{\tau} \quad \Gamma \vdash e_{i}:{ }^{{ }^{c o} \text { Inst }_{c_{i}}^{s} \vec{\tau} \rightarrow \theta \quad(1 \leq i \leq n)}}{\Gamma \vdash \text { case } e^{\prime} \text { of }\left\{c_{1} \Rightarrow e_{1}|\ldots| c_{n} \Rightarrow e_{n}\right\}: \theta} \quad \text { if } \mathrm{C}(d)=\left\{c_{1} \ldots, c_{n}\right\} \\
& \text { (corec) } \quad \frac{\Gamma, f: \vec{\sigma} \rightarrow{ }^{\mathrm{co}} d^{\imath} \vec{\tau} \vdash e:\left(\vec{\sigma} \rightarrow{ }^{\mathrm{co}} d^{\imath} \vec{\tau}\right)[\imath:=\widehat{\imath}] \quad \imath \text { pos } \vec{\sigma}}{\Gamma \vdash\left({ }^{\mathrm{co}} \text { letrec } f=e\right):\left(\vec{\sigma} \rightarrow{ }^{\mathrm{co}} d^{2} \vec{\tau}\right)[\imath:=s]} \quad \text { if } \imath \text { not in } \Gamma, \vec{\tau}
\end{aligned}
$$

Below are some programming examples that illustrate the use of co-recursive functions.

\section{Example 5.1.}

— The colist of all natural numbers starting from a given one (in the ascending order).

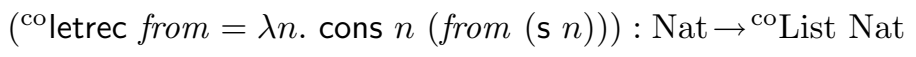

— The infinite colist consisting of zeros.

$$
\left({ }^{\mathrm{co}} \text { letrec zeros }=\text { cons o zeros }\right):{ }^{\mathrm{co}} \text { List Nat }
$$

- Concatenation of two colists. This program admits a type containing the information 
that the concatenation of two colists is in the same approximation of the colist type where the two individual colists are.

$$
\begin{aligned}
& \text { append } \equiv \quad\left({ }^{\mathrm{co}} \text { letrec append }{ }_{\mathrm{co}} \operatorname{List}^{2} \tau \rightarrow{ }^{\mathrm{co}} \operatorname{List}^{2} \tau \rightarrow{ }^{\mathrm{co}} \operatorname{List}^{2} \tau=\right.
\end{aligned}
$$

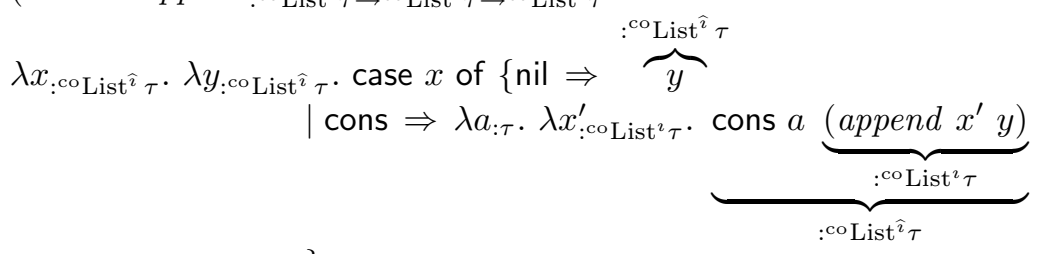

$$
\begin{aligned}
& \} \\
& \text { ) : } \quad{ }^{\mathrm{co}} \mathrm{List}^{\mathrm{s}} \tau \rightarrow{ }^{\mathrm{co}} \mathrm{List}^{\mathrm{s}} \tau \rightarrow{ }^{\mathrm{co}} \mathrm{List}^{\mathrm{s}} \tau
\end{aligned}
$$

— This program exchanges every first and second element in a given colist.

$$
\begin{aligned}
& \text { ( }{ }^{\mathrm{co}} \text { letrec } \quad \text { exch: }{ }^{\mathrm{co}} \operatorname{List} \tau \rightarrow{ }^{\mathrm{co}} \operatorname{List}^{2} \tau=\lambda l_{\text {:co } \operatorname{List} \tau} . \\
& \text { case } l \text { of }\{ \\
& \text { nil } \Rightarrow \text { nil } \\
& \text { | cons } \Rightarrow \lambda a_{: \tau} \cdot \lambda l_{: \text {co List } \tau}^{\prime} \text {.case } l^{\prime} \text { of }\{ \\
& \text { nil } \Rightarrow \text { cons } a \text { nil }
\end{aligned}
$$

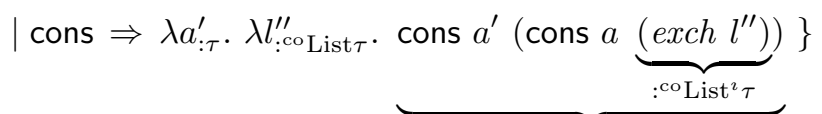

$$
\begin{aligned}
& \text { :co List }{ }^{\hat{\imath}} \tau \sqsubseteq{ }^{c o} \operatorname{List}^{\hat{\imath}} \tau \\
& \}
\end{aligned}
$$

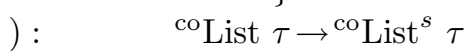

Although the exchange function does not alter the length of a colist, the type we have given to the program above is the best possible in our setting.

- As a last example, we give a program that, given a colist, computes its infinite repetition. The typability of this program is a consequence of the typing we have given for append.

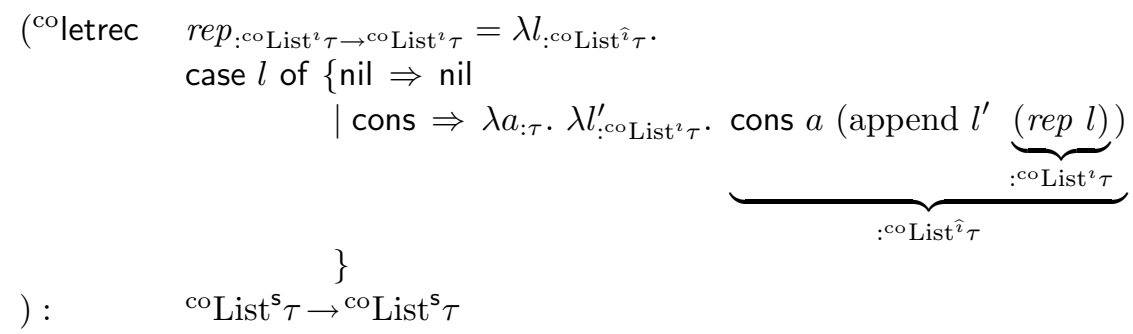

The extended $\lambda$ enjoys subject reduction and strong normalizability of typable terms just as the original $\lambda$. The proof for $\lambda$ extends readily; we leave it out for space reasons. We believe that $\lambda_{\mathcal{G}}$ extended with guarded by constructors corecursion is embeddible in

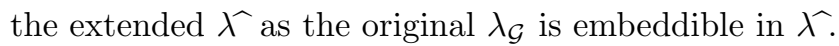




\section{Related work}

For the sake of clarity, we split existing systems into four categories: (1) based on traditional-style terminating recursors, (2) based on a fixpoint operator controlled by a syntactic guard predicate, (3) exploiting pattern matching, (4) based on a fixpoint operator controlled by an unusual typing ensuring that the recursion actually terminates, (5) relying on other type-based techniques for ensuring termination.

Comparison with (Martin-Löf 1971) and other works on traditional-style terminating recursors Most formalizations of inductive types in type theory support recursive definitions only indirectly via eliminators behaving as iterators or primitive recursors (MartinLöf 1971; Leivant 1983; Pierce et al. 1989; Pfenning and Paulin-Mohring 1990; PaulinMohring 1993; Coquand and Paulin 1990; Dybjer 1994; Geuvers 1992; Matthes 1999; Altenkirch 1999; Spławski and Urzyczyn 1999). Such systems are well-understood metatheoretically and enjoy good properties, but are hard to use in practical programming: this requires the programmer to translate all recursive definitions he would like to make into explicit definitions involving primitive recursion.

It is possible to devise similar eliminators capturing more sophisticated schemes of terminating recursion such as course-of-value iteration or course-of-value primitive recursion (Uustalu 1998; McBride 1999), but the resulting systems are even clumsier to use practically.

Comparison with (Coquand 1994) and other works relying on a fixpoint operator controlled by an external guard predicate (Coquand 1994) introduces a simple guard predicate to ensure termination of fixpoint expressions in a calculus of infinite objects. Building up on Coquand's work, (Giménez 1995) defines a more liberal guarded-by-constructors predicate for terminating corecursion and also a guarded-by-destructors predicate for terminating recursion. Giménez shows that primitive recursor expressions can be rendered as fixpoint expressions guarded by one destructor. In the opposite direction, a fixpoint expression guarded by destructors can be coded as an expression involving primitive recursors, but the translation is not uniform. The predicates defined by Giménez form the basis of the mechanism for (co)inductive types in CoQ. More recently, (Blanqui et al. 2002), building up on (Jouannaud and Okada 1997), propose another definition of the guard predicate for inductive types, that allows for yet more expressions to be typed. In a similar line of research, (Abel and Altenkirch 2002) propose a basic framework for studying and comparing the different termination conditions that have been proposed so far, focusing their attention on what conditions should be fulfilled for a checking to be sound. An application of such framework to a particular condition can be found in (Abel 2000).

One possible objection against this line of work is that the system becomes more unpredictable to the user as the complexity of the guard predicate builds up. Besides, the guard predicate remains purely syntactic, which is not appropriate for a number of applications, including separate compilation or interactive proof construction. 
Comparison with (Coquand 1992) and other works on pattern-matching (Coquand 1992) pioneers the use of pattern-matching in type theory. While pattern-matching yields leaner definitions, its proof-theoretical status in the context of dependent types remains unclear. Differently from guarded-by-destructors recursion, general pattern-matching is not a conservative improvement over primitive recursors: (Hofmann and Streicher 1994) prove the derivability of uniqueness of equality proofs in a type theory with pattern-matching, while equality proofs cannot be shown to be unique in the usual Calculus of Inductive Constructions. To our knowledge, there is no complete account of the meta-theoretical properties of pattern-matching in dependent type theory. (McBride 1999) has shown that, under the uniqueness of equality proofs as an extra axiom, pattern matching is admissible. (Giménez 1996) has remarked that in a typing system with dependent pattern matching, the computation rule used in this article for corecursive definitions only satisfies a weak form of the subject reduction property. Ongoing work on checking the termination of recursive function definitions in functional languages, see e.g. (Telford and Turner 1997; Abel and Altenkirch 2002; Giesl et al. 1998; Manoury and Simonot 1994), bears relevance for this direction of type-theoretic developments. Of particular interest for the future type-theoretic formalizations might be the recent work (Lee et al. 2001) on the size-change principle for program termination.

As to implementations, restricted forms of pattern-matching have been implemented in CoQ by (Cornes 1997) and LEGo by (Elbers 1998). Both implementations take advantage of translations to recursors. Pattern-matching has also been consistently supported in ALF and its subsequent versions, although no mechanism for termination checking was ever implemented. In order to simplify the proof engine, Agda, which is the latest incarnation of ALF, only supports a limited form of pattern-matching in which variables are only allowed to occur once in the type of a constructor. This restriction rules out, for example, inductive definitions such as equality.

Comparison with (Giménez 1998) and other works on guarded types This line of work is really about non-traditional-style terminating recursors that look like fixpoint operators, but where the computation is guaranteed to terminate by an unusual (stronger) typing system. Such system involves introducing some kind of annotations on recursive types, a notion of sub-typing enabling the transformation of such annotations, and a typing rule for the term letrec $f=e$ where the type of $f$ and the type of $e$ are marked differently. In this sense, some of the systems mentioned in this section are not far from the so called abstract interpretation techniques (Cousot and Cousot 1996), even though they are formulated from a type-theoretical point of view. The exact relation of such typing systems with respect to abstract interpretation techniques has not been studied in detail yet, and could be a subject for further research.

(Mendler 1987) was, to our knowledge, the first author to propose a formalization of inductive and coinductive types in a simply typed lambda calculus where primitive recursion and primitive corecursion were formulated in a fixpoint-like style. In Mendler's system, type annotations on the fixpoint rule correspond to type variables. (Mendler 1991) considered a system supporting only iteration and coiteration. Works that comment on these two papers include (Leivant 1990; Geuvers 1992; Uustalu and Vene 1997; Uustalu 
1998; Matthes 1998; Matthes 2002; Spławski and Urzyczyn 1999). Among these, (Leivant 1990; Geuvers 1992) were the first papers to contrast and compare traditional-style and Mendler-style terminating recursors. (Uustalu and Vene 1997; Uustalu and Vene 2002; Uustalu 1998) showed that Mendler's approach is readily generalizable for course-of-value (co)recursion (in other words, full structural (co)recursion).

(Giménez 1996) introduced an extension of the Calculus of Constructions with inductive and coinductive types, called $\mathrm{CC}^{\infty}$. The fixpoint rules in $\mathrm{CC}^{\infty}$ make use of three kinds of marks, corresponding to the stages $\infty, \iota$ and $\hat{\iota}$ using the notation of this article. This means that in $\mathrm{CC}^{\infty}$ the hat operator can not be applied to another stage, but only to stage variables. In (Giménez 1996), marks also have a second component, specifying whether the recursive type is inductive or coinductive. There is no stage polymorphism, and hence the function div of Example 2.21 can not be typed.

One of the main disadvantages of (Giménez 1996) is that it tried to tackle too many problems at once, rendering the typing calculus less clear. Among the extra features introduced in $\mathrm{CC}^{\infty}$ which are not considered in this article we may cite the following ones :

- Inductive lists are considered a subtype of coinductive ones, so that a function defined on the type ${ }^{\mathrm{co}}$ List can also be used on an element of type List.

- Annotations are placed on typing judgments, writing $x:^{s}$ List instead of $x$ : List $^{s}$. One of the original motivations for such notation was to enable the description of abstract recursion schema, where the type of the decreasing argument of the function is abstracted away using a term of the form $\lambda A$ : Set - letrec $f=\lambda x:^{s} A \cdot e$. Also, the choice of having two different universal quantifiers renders unnecessary the introduction of two types of lists (one for inductive and the other for coinductive ones) with the same constructors. On the other hand, it is less clear how an ordinal based semantics like the one proposed in this paper could be used to make sense of a term of the form $\lambda A:$ Set $\cdot \lambda x: s A \cdot e$. This is why, even though annotated quantifications were kept, the calculus in (Giménez 1996) forces $A$ in a term of the form $\lambda x:^{s} A \cdot e$ to have a recursive type at its rightmost position.

$-C^{\infty}$ is built on the top of the Calculus of Constructions, so it uses Church's style for variable binding, where the type of the abstracted variable is explicitly mentioned. Thus, types -and hence marks- may appear in the terms. As a consequence, the reduction rule for fixpoints has to replace all mark variables by the $\infty$ mark, in order to avoid having residual unbound mark identifiers in the definiens. Note that this problem is not posed in $\lambda$,, where variable binding is "à la Curry".

(Giménez 1998) introduced CCR, a different extension of the Calculus of Constructions with inductive and coinductive types, based on (not fully general) sub- and supertyping and bounded universal quantification over types. In CCR, marks are represented as type variables, like in Mendler's works, the hat operator is a type constructor, and stages are just types. Since stage variables are type variables, stage replacement corresponds just to the ordinary substitution operation of the calculus. The calculus in (Giménez 1998) was the first calculus to introduce stage polymorphism, enabling to type definitions like the function div of Example 2.21 and the stream rep of Example 5.1. The 
calculus of the present paper is very much inspired from (Giménez 1998), but replaces sub- and supertypes with approximating types, and bounded type quantification with stage quantification - the change allows the structure of stages to be uniform over all datatypes and simplifies the introduction of recursive definitions on mutually dependent inductive types. The meta-theory of CCR has not been studied yet, nor its connection with implemented extensions of a calculus of (co)inductive constructions like the system Coq. The detailed study of the main meta-theoretical properties of $\lambda$ presented in this paper can be seen as a basic stage for developing the meta-theory of an extension of the Calculus of Construction where the termination of functions is ensured by typing constraints.

(Amadio and Coupet-Grimal 1998) define a simply typed $\lambda$-calculus "à la Curry" featuring guarded coinductive types. Starting from Coquand's guardedness condition, they propose a semantics for such extension of lambda calculus based on partial equivalent relations and ordinal iteration to interpret coinductive types. From that semantics, they derive a typing rule for corecursive definitions using a mark system with three kinds of marks, that correspond in our notation to $\infty, \iota$ and $\hat{\iota}$. The semantic interpretation used to study the meta-theory of $\widehat{\lambda}$ in this article is actually an extension of the one introduced in (Amadio and Coupet-Grimal 1998) for coinductive types. Also, the need for the constraint $\imath$ pos $\sigma$ in the typing rule for recursive definitions have been already noticed by Amadio and Coupet. Their calculus introduces an extra rule enabling to treat nested fixpoint definitions of the form (letrec $f=($ letrec $g=e$ )) by reusing the mark introduced in the definition of $f$ as the mark for the variable $g$. However, the calculus described in (Amadio and Coupet-Grimal 1998) lacks of full stage polymorphism, so definitions like the function rep in 5.1 can not be typed in their system. Their calculus does not consider inductive types. On the positive side, their calculus is shown to have decidable type inference in (Bac 1998).

(Barras 1999) formalizes in CoQ a variant of Giménez' calculus $\mathrm{CC}^{\infty}$, with the purpose of proving the decidability of its typing judgment and extracting a type-checker from the proof. In Barras' calculus, inductive types are annotated with lists of marks, each one corresponding to the stages $\infty, \iota$ and $\hat{\iota}$ of our system. The use of lists of marks enables to type nested recursive function definitions like the ones considered in (Amadio and Coupet-Grimal 1998), but for inductive types. He does not consider coinductive types nor stage polymorphism. As the underlying lambda calculus is "à la Church", Barras introduces a distinguished primitive type $\mathcal{M}$ for marks, and marks are just variables of that type. Mark variables are bound in fixpoint terms, so mark erasure in fixpoint reductions corresponds just to ordinary variable substitution. The complete meta-theory of Barras' system has not been studied yet, but his system is the only mark based one for which a type-checking algorithm has been developed.

Other type-based approaches to termination analysis (Xi 2001) proposes a system of restricted dependent types, built upon DML (Xi and Pfenning 1999), to ensure program termination. In essence, his system is closely related to ours since it uses stage information to ensure termination. However, Xi's system differs from ours in its expressiveness and complexity: while we focus on the weakest calculus that uses type-based termination 
and extends other calculi based on a simple syntactic guard predicate, Xi presents a very rich system with stage arithmetic, and a notion of metric that is very useful to handle functions in several arguments. Of course, expressiveness is achieved to the detriment of simplicity and Xi's system is much more complex than ours. (Grobauer 2001) uses DML to find cost recurrences for first-order recursive definitions: a cost recurrence is an upper bound to the running time of the program w.r.t. the size of its input, and hence a witness that the recursive definition is terminating. In his work, Grobauer exploits complex features of DML, including stage arithmetic, so his techniques do not seem directly applicable to $\lambda$. Closely related is the recent work on sized types (Hughes et al. 1996; Pareto 2000; Chin and Khoo 2001).

\section{Conclusion}

We have introduced $\lambda$, a novel type system for terminating recursive functions. The salient features of $\lambda$ are its type-based approach to ensure termination through the notion of stage, and its support for stage polymorphism. The calculus is powerful enough to encode many recursive definitions rejected by existing type systems, scales up easily to mutually inductive types and supports separate compilation. In comparison to $\lambda_{\mathcal{G}}$, it has a much clearer syntax and admits a clean semantics; the strong normalization can be proved by means of a standard method. For practice, this means that $\lambda$ is less difficult to implement (implementing the guard condition of $\lambda_{\mathcal{G}}$ is error-prone) and the code written in it is more easily maintainable. This makes $\lambda^{\Upsilon}$ a good candidate base system for type theory based proof-assistants such as CoQ.

In order to validate this claim, the following steps need to be taken:

— scale up $\lambda$ to dependent types and explicit polymorphism as in (Barras 1999; Giménez 1998);

- develop type checking and type inference algorithms for $\lambda$ 个. For the purpose of proof assistants, it may be of interest to study a calculus where type annotations are given and stage annotations are inferred;

- provide mechanisms to support mutually inductive datatypes, mutually recursive definitions and recursive functions in several parameters. For the latter, some form of stage arithmetic might be needed.

In a different line of work, it may be of interest to give a precise characterization of the functions from $\mathbb{N}$ to $\mathbb{N}$ that are representable in $\lambda$.

\section{Acknowledgments}

We are grateful to our anonymous referees for the very constructive feedback we received and to Roberto Amadio for making (Bac 1998) available to us.

The work by Gilles Barthe, Maria João Frade, Luís Pinto, and Tarmo Uustalu was partially supported by the Portuguese Foundation for Science and Technology under grant no. PRAXIS XXI/C/EEI/14172/98, by the INRIA-ICCTI collaboration and by the FP5 IST project TYPES. Tarmo Uustalu received support also from the Estonian Science Foundation under grant no. 4155. 


\section{References}

Abel, A. (2000) Specification and verification of a formal system for structurally recursive functions. In T. Coquand, P. Dybjer, B. Nordström, and J. Smith (editors), Proceedings of TYPES'99, Lecture Notes in Computer Science 1956, 1-20. Springer-Verlag.

Abel, A. and Altenkirch, T. (2002) A predicative analysis of structural recursion. J. of Functional Programming 12(1), 1-41.

Altenkirch, T. (1999) Logical relations and inductive/coinductive types. In G. Gottlob, E. Grandjean, and K. Seyr (editors), Proceedings of CSL'98, Lecture Notes in Computer Science 1584, 343-354. Springer-Verlag.

Amadio, R. M. and Coupet-Grimal, S. (1998) Analysis of a guard condition in type theory (extended abstract). In M. Nivat (editor), Proceedings of FoSSaCS'98, Lecture Notes in Computer Science 1378, 48-62. Springer-Verlag.

Bac, A. (1998) Un algorithme d'inférence de types pour les types coinductifs. Memoire de DEA, École Normale Supérieure de Lyon.

Barras, B. (1999) Auto-validation d'un système de preuves avec familles inductives. PhD thesis, Université Paris 7.

Blanqui, F., Jouannaud, J.-P., and Okada, M. (2002) Inductive data type systems. Theoretical Computer Science 272(1-2), 41-68.

Chin, W.-N. and Khoo, S.-C. (2001) Calculating sized types. Higher-Order and Symbolic Computation 14(2-3), 261-300.

Coquand, T. (1992) Pattern matching with dependent types. In B. Nordström, K. Pettersson, and G. Plotkin (editors), Informal Proceedings of TYPES'92, 71-84. Dept. of Computing Science, Chalmers Univ. of Technology and Göteborg Univ. ftp://ftp.cs.chalmers.se/pub/csreports/baastad.92/proc.ps.Z.

Coquand, T. (1994) Infinite objects in type theory. In H. Barendregt and T. Nipkow (editors), Proceedings of TYPES'93, Lecture Notes in Computer Science 806, 62-78. Springer-Verlag.

Coquand, T. and Paulin, C. (1990) Inductively defined types (preliminary version). In P. MartinLöf and G. Mints (editors), Proceedings of COLOG'88, Lecture Notes in Computer Science 417, 50-66. Springer-Verlag.

Cornes, C. (1997) Conception d'un langage de haut niveau de representation de preuves: Récurrence par filtrage de motifs; Unification en présence de types inductifs primitifs; Synthèse de lemmes d'inversion. PhD thesis, Université de Paris 7.

Cousot, P. and Cousot, R. (1996) Abstract interpretation. ACM Computing Surveys 28(2), 324-328.

Dybjer, P. (1994) Inductive families. Formal Aspects of Computing 6(4), 440-465.

Elbers, H. (1998) Connecting formal and informal mathematics. PhD thesis, Technische Universiteit Eindhoven.

Geuvers, H. (1992) Inductive and coinductive types with iteration and recursion. In B. Nordström, K. Pettersson, and G. Plotkin (editors), Informal Proceedings of TYPES'92, 193217. Dept. of Computing Science, Chalmers Univ. of Technology and Göteborg Univ. ftp://ftp.cs.chalmers.se/pub/cs-reports/baastad.92/proc.ps.Z.

Giesl, J., Walther, C., and Brauburger, J. (1998) Termination analysis for functional programs. In W. Bibel and P. Schmitt (editors), Automated Deduction: A Basis for Applications, Vol. 3: Applications, Applied Logic Series 10, 135-164. Kluwer Academic Publishers.

Giménez, E. (1995) Codifying guarded definitions with recursion schemes. In P. Dybjer and B. Nordström (editors), Proceedings of TYPES'94, Lecture Notes in Computer Science 996, 39-59. Springer-Verlag. 
Giménez, E. (1996) A calculus of infinite constructions and its application to the verification of reactive systems. PhD thesis, Ecole Normale Supérieure de Lyon.

Giménez, E. (1998) Structural recursive definitions in Type Theory. In K. G. Larsen, S. Skyum, and G. Winskel (editors), Proceedings of ICALP'98, Lecture Notes in Computer Science 1443, 397-408. Springer-Verlag.

Grobauer, B. (2001) Cost recurrences for DML programs. In Proceedings of ICFP'01, SIGPLAN Notices 36(10), 253-264. ACM Press.

Hofmann, M. and Streicher, T. (1994) The groupoid model refutes uniqueness of identity proofs. In Proceedings of LICS'94, 208-212. IEEE CS Press.

Hughes, J., Pareto, L., and Sabry, A. (1996) Proving the correctness of reactive systems using sized types. In Proceedings of POPL'96, 410-423. ACM Press.

Jouannaud, J. P. and Okada, M. (1997) Abstract data type systems. Theoretical Computer Science 173(2):349-391.

Lee C.-S., Jones, N. D. and Ben-Amram, A. M. (2001) The size-change principle for program termination. In Proceedings of POPL'01, SIGPLAN Notices 36(3), 81-92. ACM Press.

Leivant, D. (1983) Reasoning about functional programs and complexity classes associated with type disciplines. In Proceedings of FOCS'83, 460-469. IEEE Computer Society Press.

Leivant, D. (1990) Contracting proofs to programs. In P. Odifreddi (editor), Logic and Computer Science, APIC Studies in Data Processing 31, 279-327. Academic Press.

Luo, Z. (1994) Computation and Reasoning: A Type Theory for Computer Science, Int. Series of Monographs in Computer Science 11. Clarendon Press.

Manoury, P. and Simonot, M. (1994) Automatizing termination proofs of recursively defined functions. Theoretical Computer Science 135(2), 319-343.

Martin-Löf, P. (1971) Hauptsatz for the intuitionistic theory of iterated inductive definitions. In J. E. Fenstad (editor), Proceedings of 2nd Scandinavian Logic Symp., Studies in Logic and the Foundations of Mathematics 63, 179-216. North-Holland Publ. Co.

Matthes, R. (1998) Extensions of System F by Iteration and Primitive Recursion on Monotone Inductive Types. PhD thesis, Fachbereich Mathematik, Ludwig-Maximilians-Universität München.

Matthes, R. (1999) Monotone fixed-point types and strong normalization. In G. Gottlob, E. Grandjean, and K. Seyr (editors), Proceedings of CSL'98, Lecture Notes in Computer Science 1584, 298-312. Springer-Verlag.

Matthes, R. (2002) Tarski's fixed-point theorem and lambda calculi with monotone inductive types. Synthese 133(1), 107-129.

McBride, C. (1999) Dependently Typed Functional Programs and Their Proofs. PhD thesis, Laboratory for Foundations of Computer Science, Dept. of Computer Science, Univ. of Edinburgh.

Mendler, N. P. (1987) Recursive types and type constraints in second-order lambda-calculus. In Proceedings of LICS'87, 30-36. IEEE Computer Society Press.

Mendler N. P. (1991) Inductive types and type constraints in the second-order lambda-calculus. Annals of Pure and Applied Logic 51(1-2), 159-172.

Nordström, B., Petersson, K. and Smith, J. (1990) Programming in Martin-Löf's Type Theory: An Introduction, Int. Series of Monographs on Computer Science 7, Clarendon Press.

Pareto, L. (2000) Types for crash prevention. PhD thesis, Chalmers Univ. of Techn., Göteborg. Paulin-Mohring, C. (1993) Inductive definitions in the system Coq: Rules and properties. In M. Bezem and J. F. Groote (editors), Proceedings of TLCA'93, Lecture Notes in Computer Science 664, 328-345. Springer-Verlag. 
Pfenning, F. and Paulin-Mohring, C. (1990) Inductively defined types in the calculus of constructions. In M. Main, A. Melton, M. Mislove, and D. Schmidt (editors), Proceedings of MFPS'89, Lecture Notes in Computer Science 442, 209-228. Springer-Verlag.

Pierce, B., Dietzen, S. and Michaylov, S. (1989) Programming in higher-order typed lambdacalculi. Technical Report CMU-CS-89-111, School of Computer Science, Carnegie-Mellon Univ.

Spławski, Z. and Urzyczyn, P. (1999) Type fixpoints: Iteration vs. recursion. In Proceedings of ICFP'99, SIGPLAN Notices 34(9), 102-113. ACM Press.

Telford, A. and Turner, D. (1997) Ensuring streams flow. In M. Johnson (editor), Proceedings of AMAST'97, Lecture Notes in Computer Science 1349, 509-523. Springer-Verlag.

Uustalu, T. (1998) Natural Deduction for Intuitionistic Least and Greatest Fixedpoint Logics, with an Application to Program Construction. PhD thesis (Dissertation TRITA-IT AVH 98:03), Dept. of Teleinformatics, Royal Inst. of Technology, Stockholm.

Uustalu, T. and Vene, V. (1997) A cube of proof systems for the intuitionistic predicate $\mu, \nu$ logic. In M. Haveraaen and O. Owe (editors), Proceedings of NWPT'96, Research Report 248, Dept. of Informatics, University of Oslo, 237-246.

Uustalu, T. and Vene, V. (2002) Least and greatest fixedpoints in intuitionistic natural deduction. Theoretical Computer Science 272(1-2), 315-339.

Xi, H. and Pfenning, F. (1998) Eliminating array bound checking through dependent types. In Proceedings of PLDI'98, SIGPLAN Notices 33(5), 249-257. ACM Press.

Xi, H. and Pfenning, F. (1999) Dependent types in practical programming. In Proceedings of POPL'99, 214-227. ACM Press.

Xi, H. (2001) Dependent types for program termination verification. In Proceedings of LICS'01, 231-242. IEEE CS Press. 